HWR 02-010

\title{
Stochastic Fusion of Information for Characterizing and Monitoring the Vadose Zone
}

\author{
by \\ Tian-Chyi J. Yeh \\ Jirka Šimůnek \\ And \\ Martinus Th. Van Genuchten
}

Department of Hydrology and Water Resources University of Arizona

Tucson AZ 85721

March 2002 


\title{
STOCHASTIC FUSION OF INFORMATION FOR CHARACTERIZING AND MONITORING THE VADOSE ZONE
}

\author{
Tian-Chyi J. Yeh ${ }^{1}$, Jirka Šimůnek ${ }^{2}$ and Martinus Th. van Genuchten ${ }^{2}$ \\ ${ }^{1}$ Department of Hydrology and Water Resources, The University of Arizona, Tucson, Arizona \\ 85721.yeh@hwr.arizona.edu. \\ ${ }^{2}$ George E. Brown, Jr. Salinity Laboratory, USDA, ARS, Riverside, California 92507-4617.
}

\begin{abstract}
Inverse problems for vadose zone hydrological processes are often being perceived as illposed and intractable. Consequently, solutions to inverse problems are often subject to skepticism. In this paper, using examples, we elucidate difficulties associated with inverse problems and the prerequisites for such problems to be well-posed so that a unique solution exists. We subsequently explain the need of a stochastic conceptualization of the inverse problem and, in turn, the conditional-effective-parameter concept. This concept aims to resolve the ill-posed nature of inverse problems for the vadose zone, for which generally only sparse data are available. Next, the development of inverse methods for the vadose zone, based on a conditional-effective-parameter concept, is explored, including cokriging, the use of a successive linear estimator, and a sequential estimator. Their applications to the vadose zone inverse problems are subsequently examined, which include hydraulic/pneumatic and electrical resistivity tomography surveys, and hydraulic conductivity estimation using observed pressure heads, concentrations, and arrival times. Finally, a stochastic information fusion technology is presented that assimilates information from unsaturated hydraulic tomography and electrical resistivity tomography. This technology offers great promise to effectively characterize heterogeneity, to monitor processes in the vadose zone, and to quantify uncertainty associated with vadose zone characterization and monitoring.
\end{abstract}




\section{Introduction}

Knowledge of the unsaturated soil hydraulic properties is indispensable for successful predictions of water flow and solute transport in the vadose zone. A variety of laboratory and field methods are currently available for direct and indirect evaluation of the unsaturated hydraulic conductivity, $K$, as a function of the pressure head, $h$, and/or the water content, $\theta$. Popular laboratory methods include the one- and multi-step outflow methods (Kool et al., 1985; van Dam et al., 1994), the upward infiltration method (Hudson et al., 1996), and the evaporation method (Gardner and Miklich, 1962, Šimůnek et al., 1998). Popular field methods include the instantaneous profile method (Hillel et al., 1972), various unit-gradient type approaches, sorptivity methods following ponded infiltration, and the crust method based on steady water flow. While existing field methods are relatively simple in concept, these direct measurement methods have a number of limitations that restrict their use in practice. For example, most methods are very timeconsuming to execute because of the need to adhere to relatively restrictive initial and boundary conditions. This is especially true for field gravity-drainage experiments involving medium- and fine-textured soils. While most of the above methods are widely used and accepted, questions of parameter identifiability and their uniqueness for particular methods are still often raised.

While various laboratory and field methods for evaluating soil hydraulic properties are relatively well established, several major problems remain. Most laboratory methods are applied to samples ranging from $100 \mathrm{~cm}^{3}$ to about $500 \mathrm{~cm}^{3}$. The scale of field methods generally does not extend beyond a plot of $1 \mathrm{~m}^{2}$ and depths of one to several meters. There is an urgent need to develop methods that characterize hydraulic properties of the vadose zone on a much larger scale. For large-scale investigations, recently developed geophysical methods (such as electrical resistivity tomography, ERT; ground penetrating radars, GPR; Binley et al., 2001. Daily et al., 1992) for characterizing and monitoring the vadose zone, coupled with geostatistically based inverse methods (Hughson and Yeh, 2000, and Yeh et al., 2002) appear to be promising.

In this paper we use simple examples to first examine conditions required to make inverse problems well posed for both steady state and transient flow through homogeneous media. Conditions for flow through simplified heterogeneous media are subsequently explored, and stochastic conceptualizations of inverse problems for the vadose zone are introduced. Next, we describe several geostatistically based inverse approaches, including cokriging, a successive linear estimator, and a sequential conditioning method. Various techniques such as hydraulic tomography and electrical resistivity tomography are subsequently discussed to enhance vadose zone characterization and monitoring using the geostatistically based inverse approaches.

Finally, we introduce a stochastic fusion of information concept to assimilate information from soil physics, hydrology, geophysics, and geology for characterizing and monitoring the vadose zone. Preliminary results of the information fusion technology are presented. We hope that our discussions will lead to better-designed laboratory and field experiments, as well as to vigorous research and development of integrative inversion approaches for characterizing and monitoring the vadose zone. 


\section{Inverse Problem in Subsurface Hydrology-A Well-Posed and a Ill-Posed Problem}

Movement of water in variably saturated porous media under isothermal conditions, neglecting the gas phase, may be described using a modified form of the Richards equation

$$
\omega S_{s} \frac{\partial h}{\partial t}+\frac{\partial \theta}{\partial t}=\nabla \cdot[K(h) \nabla(h+z)]
$$

where $h$ is the pressure head, which is positive when soil is fully saturated and negative when the soil is partially saturated, $t$ is time, $\theta$ stands for the volumetric moisture content, and $z$ is the positive upward vertical coordinate. The term $S_{s}$ represents specific storage and $\omega$ a transitioning parameter that is one when $h$ is greater than or equal to zero and zero when $h$ is negative. To describe the $\theta$ - $h$ relationship of unsaturated media, van Genuchten's (1980) model is often assumed:

$$
\theta(h)=\theta_{r}+\left(\theta_{s}-\theta_{r}\right)\left(1+|\alpha h|^{n}\right)^{-m}
$$

where $\|$ is the absolute value, $\theta_{s}$ is the saturated moisture content, $\theta_{r}$ is the moisture content at residual saturation and $\alpha, n$, and $m$ are fitting shape parameters with $m=1-1 / n$. We further assume that the unsaturated hydraulic conductivity function, $K(h)$, follows Mualem's pore-size distribution model (van Genuchten, 1980), i.e.,

$$
K(h)=K_{s} \frac{\left(1-(\alpha|h|)^{n-1}\left[1+(\alpha|h|)^{n}\right]^{-m}\right)^{2}}{\left[1+(\alpha|h|)^{n}\right]^{m / 2}}
$$

where $K_{s}$ is the locally isotropic saturated hydraulic conductivity. Hereafter, (2) and (3) are referred to as the VG model.

If we consider only flow through fully saturated media, (1) simplifies to

$$
\nabla \cdot\left(K_{s} \nabla \phi\right)=S_{s} \frac{\partial \phi}{\partial t}
$$

where $\phi=h+z$ is the hydraulic head. Generally, the boundary conditions associated with equations (1) and (4) are given as: a) $K(h) \nabla \phi=n \cdot q^{*}$ at $\Omega_{1}$, where $n$ is a normal unit vector and $q^{*}$ a specified specific discharge, and b) $\phi(x, y, z)=\phi^{*}$ at $\Omega_{2}$, where $\phi^{*}$ is the specified hydraulic head. The initial condition is given as $\phi(x, y, z, 0)=\phi^{*}(x, y, z, 0)$ at $t=0$ and all locations.

If we define $K_{s}, S_{s}, \alpha, n, \theta_{s}$, and $\theta_{r}$ as parameters or primary variables, then $\phi, h$, and $\theta$ are state variables, secondary variables or system responses. A forward problem refers to solving the flow equation for the hydraulic head or moisture distribution in time and space with known $K_{s}, S_{s}, \alpha, n, \theta_{s}$, and $\theta_{r}$, and for given initial and boundary conditions. In other words, the forward problem predicts the behavior or response of a system based on known system parameters, initial and boundary conditions. On the other hand, an inverse problem refers to determinating values of the $K_{s}, S_{s}, \alpha, n, \theta_{s}$, and $\theta_{r}$ parameter from information about excitations to the system and its response to those excitations (e.g., in terms of observed $\phi, h$, or/and $\theta$ values). In other words, an inverse solution seeks parameters values that can reproduce the observed head, flux or/and moisture content distributions in time and space.

A forward problem is well posed if the parameters, initial and boundary conditions are completely specified in the solution domain so that the problem can have a unique solution. It is 
ill posed and has an infinite number of solutions otherwise. A well-posed problem however does not necessarily warrant a solution. For instance, a well-posed forward problem for variably saturated flow may still encounter convergence and stability issues due to inadequate solution techniques.

An inverse problem is ill posed if there is no unique solution to the inverse problem. In the following, we will examine conditions necessary for an inverse problem to be well posed for flow in homogeneous and heterogeneous media for both steady state and transient flow, as well as for saturated and unsaturated conditions.

\subsection{Homogeneous Porous Media}

2.1.1. Steady-state saturated flow. Consider one-dimensional, steady-state, saturated flow through a homogeneous soil column. If the specific discharge (flux boundary condition) and the hydraulic head distribution or gradient along the column are specified, then the hydraulic conductivity of the column can be determined uniquely using Darcy's Law: $K_{s}=-q /(d \phi / d x)$. The inverse problem is ill posed, otherwise. That is to say, in order to have a unique inverse solution to a steady saturated flow problem, the spatial hydraulic head distribution - from which the gradient can be determined - and a flux boundary condition must be specified beforehand.

2.1.2 Transient saturated flow. The inverse problem becomes more complex during transient saturated flow. The governing flow equation for a one-dimensional saturated flow problem is given by

$$
K_{s} \frac{\partial^{2} \phi}{\partial x^{2}}=S_{s} \frac{\partial \phi}{\partial t}
$$

Notice that (5) can be written as a diffusion equation where the diffusion coefficient is the ratio of $K_{s}$ to $S_{s}$. In general it is impossible to identify $K_{s}$ to $S_{s}$ uniquely based on hydraulic head measurements only. Approaches to circumvent this problem however exist. For example, integrating the above equation from $x=0$ to $x=L$ yields

$$
\left.K_{s} \frac{\partial \phi}{\partial x}\right|_{x=L}=S_{s} \frac{\partial}{\partial t}\left[\int_{x=0}^{x=L} \phi(x, t)\right]+\left.K_{s} \frac{\partial \phi}{\partial x}\right|_{x=0}
$$

The term on the left-hand side of (6) is the specific discharge evaluated at $x=L$. The first term on the right-hand side of (6) represents the change in storage, while the second term denotes the specific discharge flowing into the column at $x=0$. Sum of these two terms results in the specific discharge at the end of the column, $q(L, t)$. Hence, we can rewrite (6) as

$$
-\left.K_{s} \frac{\partial \phi(L, t)}{\partial x}\right|_{x=L}=q(L, t)
$$

In (7), the hydraulic head at $x=L$ is a function of $t$, and the specific discharge at the end of the

column hence is no longer a constant but varies with time. Under such transient conditions, a unique value for $K_{s}$ can be derived provided that the specific discharge, $q(L, t)$, is known and the gradient, $\partial \phi / \partial x$, at $x=L$ and at the time when $q(L, t)$ can be measured. Otherwise, the inverse problem is ill posed. Similarly, (6) shows that in order to uniquely determine the specific storage term, $S_{s}$, the rate of change in the head over the length of the column and the difference between inflow and outflow must be known. Alternatively, the spatial distribution of the hydraulic head for at least at two time steps and the hydraulic conductivity must be given in order to determine 
uniquely the specific storage. In fact, if the hydraulic head spatial distributions at three time steps and two different specific discharge values at one of the boundary are known, one can form two mutually independent equations using (6). The equations can then be solved simultaneously to uniquely determine $K_{s}$ and $S_{s}$. An alternative to this approach is to first determine $K_{s}$ using the late time (steady-state) hydraulic head spatial distribution and specific discharge information. The hydraulic head distribution information at $t=0$ and one transient time step may be then used to determine $S_{s}$. The inverse problem in that case is well posed.

2.1.3. Steady-state unsaturated flow. The hydraulic conductivity being a function of the pressure head or moisture content generally augments difficulties for inverse problems for flow through unsaturated porous media. Consider steady one-dimensional vertical flow in an unsaturated soil column and assume Darcy's law to be valid for the flow process:

$$
q=-K(h)[d h / d z+1]
$$

In this case, the pressure head, $h$, along the column must be specified in addition to the specific discharge so that the unsaturated hydraulic conductivity at the given pressure head can be determined. Knowledge of the pressure head distribution allows an accurate evaluation of the pressure gradient. Based on this principle, a unit gradient approach determines the unsaturated hydraulic conductivity. Under a unit gradient condition, the specific discharge is equal to the unsaturated hydraulic conductivity at the given pressure head. Since the hydraulic conductivity varies with the pressure head, several steady unit gradient flows situations with different flow rates must be created in order to determine accurately the shape of the hydraulic conductivity function. The number of steady flows nevertheless can be reduced if we prescribe a certain relationship between $K$ and $h$. For example, if we assume that $K(h)=K_{s} \exp (\beta h)$, where $K_{s}$ was previously defined and $\beta$ is a pore-size distribution parameter (Gardner, 1958), we have

$$
q=-K_{s} \exp (\beta h)
$$

In this case, two independent equations are required to uniquely determine the value of $K_{s}$ and $\beta$, implying that at least two, steady unit-gradient type flow experiments with different pressure head values must be carried out. The problem otherwise will be ill posed. Similarly, if the VG model (Eq. 3) is used, which has three parameters $\left(K_{s}, \alpha\right.$, and $\left.n\right)$, three unit gradient conditions with different flow rates must be implemented such that three independent equations exist to warrant a unique solution. When the experiment is not carried out under a unit gradient condition, only one flow rate experiment is required with pressure heads and pressure head gradients known at at least two and three locations for the Gardner and VG models, respectively. Two or three mutually independent equations thus can be established. Consequently, the inverse problem becomes well posed although the resultant nonlinear equations may be still difficult to solve.

2.1.4. Transient unsaturated flow. For transient unsaturated flow problem, after integrating the Richards equation (1) over the length of the soil column (from $z=0$, the bottom of the column, to $z=L$, the top of the column), we have

$$
-\left.K(h)\left[\frac{\partial h}{\partial z}+1\right]\right|_{z=0}=\frac{\partial}{\partial t}\left[\int_{z=0}^{z=L} \theta(z, t)\right]-\left.K(h)\left[\frac{\partial h}{\partial z}+1\right]\right|_{z=L}
$$

The term on the left-hand side of $(10)$ is the outflow evaluated at $z=0$. The first term on the right-hand side of (10) denotes the change in storage while the second term is the specific 
discharge flowing into the column at $z=L$. The sum of the two terms on the right-hand side of (10) yields the specific discharge at the end of the column, $q(0, t)$.

$$
-\left.K(h)\left[\frac{\partial h}{\partial z}+1\right]\right|_{z=0}=q(0, t)
$$

Equation (11) suggests that the pressure head, the pressure gradient, and the discharge at $z=0$ at a specified time must be known beforehand in order to uniquely define $K(h)$ at the given pressure head. Again, the minimum number of times when these measurements must be specified will depend on the form of the invoked conductivity model. For instance, if the VG model with three unknown parameters $\left(K_{s}, \alpha\right.$, and $\left.n\right)$ is used, at least three different discharge rates and pressure head profiles at three times are needed to create three independent equations. This scenario actually forms the basis of the well-known instantaneous profile method (Rose et al., 1965; Watson, 1966).

Knowledge of the pressure head and moisture content distributions along the column at two specified times allows evaluation of the pressure head gradient and the change in moisture content along the length of the column. However, this is not sufficient to make the inverse problem of (10) well posed. Since the unsaturated hydraulic conductivities $K(h)$ at $z=0$ and $z=$ $L$ remains unknown, the number of unknowns is greater than the number of independent equations. Additional information about the pressure head and moisture content distributions at different times does not help because more unknowns (i.e., $K(h)$ at different $h$ 's) are introduced. Consequently, to resolve the ill-posedness issue, in addition to the head and moisture content distributions at different times, one of the boundary fluxes in (10) at different times (assuming the magnitudes are non-zero) must be specified. Notice that if measurements of both the pressure head and the moisture content at a point in a homogeneous soil column at different times are available, the water retention curve $\theta(h)$ of the medium can be constructed. If we assume that the parameter values for the retention curve are representative of the relative conductivity curve based on the VG model, the relative conductivity/pressure head relationship can be derived without inverting the flow model.

The discussions above illustrate a simple fact: if sufficient information is available to yield enough independent equations, the inverse problem is always well posed and should have a unique solution. Specifically, for steady state saturated flow problems, the pressure head distribution and the flux must be specified to uniquely determine $K_{s}$. In the case of transient saturated flow, both $K_{s}$ and $S_{s}$ are unknowns to be determined. The flux and the pressure head distributions at three different times are then the necessary conditions for ensuring uniqueness of the inverse solution. Similarly, for unsaturated steady flow problems, knowledge of the pressure head distributions for at least one steady flux, depending upon the presence of a unit gradient condition, can ensure a unique solution. For transient unsaturated flow problems, in addition to knowledge of the pressure head distribution at different times, information about the moisture content will enhance the inverse solution, while information of the flux is again mandatory.

A well-posed problem, however, may still fail to yield a solution because of limitations of the adopted solution technique. Furthermore, the above discussions are restricted to inversions based on mathematical models, which assume one to one relationship between the hydraulic property and the hydraulic response of a system in both forward and inverse operations. The inversion of hydraulic properties from actual laboratory and field experiments is generally far 
more complicated due to other factors. These factors include the representativeness of the mathematical models (model error), the ability to measure detailed $h$ and $\theta$ distributions and fluxes (lack of information), precision of numerical models and computational devices (numerical errors), and noise in the measurements (measurement errors). Additionally, in experiments for the inverse problem, consistency between measurements and representative elementary volume (REV) scale must be met (i.e., the measurements must be representative of the flow behavior over a REV.) The material property identified does not otherwise represent the REV. Conversely, the material property identified based on representative hydraulic head measurements can not depict heterogeneity smaller than the REV. A large number of spatial measurements of the pressure head and the moisture content may well overcome the difference in scale between the REV and the measurements. Many recent investigations by soil physicists (Toorman et al., 1992; Zurmühl, 1996; Šimůnek et al., 1997, 1998) have developed practical approaches to circumvent these difficulties. Nevertheless, the well-posedness conditions discussed above are prerequisites for having a unique solution to the inverse problem. These prerequisites must be considered a priori in the design of field and laboratory experiments for the inverse problem.

\subsection{Heterogeneous Porous Media.}

Having spatially varying hydraulic properties compounds the difficulties of inverse problems for flow through heterogeneous porous media. Still, the principles discussed above for homogeneous media remain applicable.

2.2.1. Steady-state saturated flow. Consider steady-state saturated flow through a soil column consisting of several blocks having different hydraulic conductivity values. If the specific discharge and the pressure head distributions, and in turn the hydraulic gradients, along the column are known exactly, the inverse problem is well posed and a unique solution to the inverse problem will exist. Again, the hydraulic head measurement is assumed to be representative of the average hydraulic head over the REV for each homogeneous material in the column.

Now, examine steady saturated flow in a heterogeneous soil column (Figure 1 ) in which we know only the specific discharge, $q$, pressure heads at two locations, namely, $h_{0}$ and $h_{3}$, and the size of each hydraulic conductivity block. Because the flow is steady and one-dimensional, the specific discharge must be the same along the column based on the continuity equation. Assuming the flow follows Darcy's law, we can formulate the following relationships:

$$
-K_{s 1}\left(h_{1}-h_{0}\right)=q \Delta x,-K_{s 2}\left(h_{2}-h_{1}\right)=q \Delta x,-K_{s 3}\left(h_{3}-h_{2}\right)=q \Delta x
$$

where $\Delta x$ is the block length, which is assumed constant. As shown in (12), we have five unknowns (i.e., $K_{s 1}, K_{s 2}, K_{s 3}, h_{1}$ and $h_{2}$ ) and three equations. Consequently, the problem is ill posed and there is no unique solution when only $q, h_{0}$ and $h_{3}$ are measured. Figure 1 shows several possible head distributions (dashed lines) that may produce the same discharge and the same head values at $x_{0}$ and $x_{3}$. A large number of such head distributions exist although they are bounded by the two limiting head distributions indicated by solid lines: one at the top and one at the bottom. Each head distribution (dashed line) corresponds to a specific hydraulic conductivity distribution along the column. Accordingly, a large number of possible conductivity values can satisfy (12), but they must be bounded by a maximum value, $K_{\max }=-q /(d h / d x) \approx \infty$ since $d h / d x$ 
approaches zero (without reaching zero), and a minimum value, $K_{\min }=-q /\left[\left(h_{1}{ }^{-}{ }^{-} h_{0}\right) / \Delta x\right]$. Notice that it is negative indicating flow from left to right and $h_{1}^{-}$is the minimum head at $x_{1}$ (sign indicate minimum possible value at $x_{1}$ ), which is slightly greater than the $h_{3}$ at $x_{3}$ so that a nonzero gradient exists and the given $q$ is maintained.

While a large number of conductivity values satisfy (12), the effective hydraulic conductivity of the heterogeneous column is unique. Specifically, when using the heads $h_{0}$ and $h_{3}$, one can define an ensemble mean hydraulic head distribution (the average of all possible heads) between $x_{0}$ and $x_{3}$ (middle solid line in Figure 1), which then implies that the mean gradient is known. If the specific discharge is also given, the solution to the effective hydraulic conductivity can be derived explicitly, i.e., the harmonic mean of $K_{s 1}, K_{s 2}$, and $K_{s 3}$. This effective hydraulic conductivity is, however, not the true hydraulic conductivity value of each block but the hydraulic conductivity for an equivalent homogeneous medium that can reproduce the specific discharge, and yields the mean pressure head distribution.

Now, let us assume that $h_{1}$ is also specified in the flow system of Figure 1. Possible head distributions corresponding to this case are shown in Figure 2. Accordingly, possible hydraulic conductivity values associated with the heads are bounded by a maximum value, $K_{\max }=$ $q /(d h / d x) \approx \infty$ since $d h / d x$ of the upper bound of the head distribution approaches zero, and a minimum value, $K_{\min }=-q /\left[\left(h_{2}^{-}-h_{1}\right) / \Delta x\right]$. Notice that $h_{2}{ }^{-}$is the minimum head at $x_{2}$, which is slightly greater than the $h_{3}$ at $x_{3}$ so that the given $q$ can be maintained. While this inverse problem remains ill posed, the range of possible head and hydraulic conductivity values becomes narrower as compared with those in Figure 1, indicating that additional information reduces the range of possible head and hydraulic conductivity values. This is true in this case as long as $h_{1}$ is not equal to the maximum of the possible $h_{1}$ values in Figure 1.

Likewise, if we know the value of $K_{s 2}$, we then have the gradient between locations $x_{1}$ and $x_{2}$. The range of possible head distributions (Figure 3 ) is thus smaller than in Figure 1 because the distributions of in $K_{s 1}$ and $K_{s 3}$ must be consistent with the gradient within the material with $K_{s 2}$. Therefore, possible hydraulic conductivity values are constrained. They are bounded by a maximum value, $K_{\max }=-q /(d h / d x) \approx \infty$, and a minimum value, $K_{\min }=-q \Delta x /\left[h_{3}-\right.$ $h_{2}$ ] where $h_{2}=\left(h_{0}{ }^{+}+q \Delta x / K_{s 2}\right)$. Note that the $h_{0}{ }^{+}$is the maximum head at $x_{1}$, which is slightly smaller than $h_{0}$ so that a non-zero gradient exists between $x_{0}$ and $x_{1}$ and the given $q$ is maintained. Although the range of possible values for the hydraulic conductivity is smaller, comparing with Figures 1 and 2, the inverse problem remains ill posed. Again, this is true as long as the given $K_{s 2}$ is not equal to the $K_{\min }$ in Figures 1 and 2. In this case, specifying only two of the five unknowns (e.g., $h_{1}$ and $h_{2}$ ) in (12) can make the inverse problem well posed.

Based on the above rudimentary reasoning, it is apparently impossible to determine the exact values for $K_{s 1}, K_{s 2}$ and $K_{s 3}$ in Figure 1 if information about head or hydraulic conductivity is fragmentary. Neither can the values of $K_{s 2}$ and $K_{s 3}$ in Figure 2, nor the values of $K_{s 1}$ and $K_{s 3}$ in Figure 3, be determined. Under this circumstance, the only viable solution to the inverse problem is to determine a conditional effective conductivity for the sections between $x_{1}$ and $x_{3}$ if $h_{1}$ is prescribed. The conditional effective hydraulic conductivity thus will be a harmonic mean of $K_{s 2}$ and $K_{s 3}$, which can reproduce the observed $h_{1}$ and $h_{3}$ values and yield a mean head distribution (dark line) between $x_{1}$ and $x_{3}$. Similarly, the effective hydraulic conductivity values for the first and third blocks in Figure 3 are the ones that can produce mean head distributions between $x_{0}$ and $x_{1}$ given $h_{0}$, and between $x_{2}$ and $x_{3}$ given $h_{3}$. Such effective conductivity values 
will also yield a mean head distribution (dark line) between $x_{1}$ and $x_{2}$, with a gradient necessary to produce the specified $q$ with the given $K_{s 2}$. The inverse problem for identifying the effective conductivity in these cases thus becomes well posed and can have a unique solution. According to this conditional effective approach, a well-posed inverse problem does not imply that one can identify the exact parameter values of the flow system but the most likely parameter values conditioned on given observations.

2.2.2. Transient saturated flow. For transient saturated flow in a medium with spatial varying $K_{s}$ and $S_{s}$, difficulties in the inversion are augmented by the fact that additional unknowns, $S_{s}$ 's, are to be determined. Nevertheless, the inverse problem will be well posed if both spatial and temporal pressure head distributions along the column and the fluxes (as discussed in the homogeneous case) are fully specified. For example, suppose that $K_{s}$ values of each block are determined first using a given steady state head distribution and a boundary flux as described in previous section. To uniquely identify the $S_{s}$ value of each block, temporal head variations at at least two different times in each block must be given. This implies that the $S_{s}$ value of a block can be determined only if the head change within the block is specified.

2.2.3. Steady-state unsaturated flow. Consider one-dimensional steady unsaturated infiltration into a column consisting of three layers. The unsaturated hydraulic conductivity of each layeris assumed to be described by the exponential model, $K(h)=K_{s} \exp (\beta h)$, and the thickness of the layer is constant, $\Delta z$. Therefore, we have three equations:

$$
\begin{aligned}
& K_{s 1} \exp \left[\beta_{1} h(z)\right]\left[\frac{d h(z)}{d z}+1\right]=-q \quad z \in\left(z_{0}, z_{1}\right) \\
& K_{s 2} \exp \left[\beta_{2} h(z)\right]\left[\frac{d h(z)}{d z}+1\right]=-q \quad z \in\left(z_{1}, z_{2}\right) \\
& K_{s 3} \exp \left[\beta_{3} h(z)\right]\left[\frac{d h(z)}{d z}+1\right]=-q \quad z \in\left(z_{2}, z_{3}\right)
\end{aligned}
$$

Each of these equations has two unknowns $\left(K_{s}\right.$ and $\left.\beta\right)$ if $q$ and $h$ are specified. Note that for unsaturated flow $h$ varies nonlinearly within each material. Consequently, having a detailed $h$ distribution for one steady flow rate is necessary to allow specification of $h$ and $d h / d z$ values at different $z$ 's such that two independent equations can be formulated for each material. A unique solution of $K_{s}$ and $\beta$ of each medium is then possible. If only one $h$ in each material is known, imposing two different flow rates is essential so that two independent equations for each material exist. For example, one may determine $K_{s}$ of each medium first, using a detailed $h$ distribution during a fully saturated flow as discussed previously. Values of the $\beta$ s are subsequently identified from a steady-state $h$ distribution and the flux during unsaturated flow. When the VG model with three parameters $\left(K_{s}, \alpha\right.$, and $\left.n\right)$ is used, spatial $h$ distributions at three different flow rates (e.g., one saturated and two unsaturated experiments) will ensure existence of three independent equations for each block. Thus, they can lead to a unique solution.

To verify this concept, Figures $4 \mathrm{a}$ and b show the true values of $K_{s}$ and $\alpha$ (after removing their means) in a synthetic soil profile with 20 layers, respectively, as well as those estimated. The estimated values were obtained using the SLE approach (see Section 5.2) with one pressure head measurement in each layer for one steady unsaturated flux situation. This inverse problem is ill-posed since each layer has two unknowns, $K_{s}$ and $\alpha$ but only one pressure head value, and 
thus only one independent equation. The estimates hence deviate from the true ones although they resemble the true fields. If one fully saturated and one unsaturated steady flux experiment are conducted, two different pressure head values and thus two independent equations become available for each layer. The inverse problem then becomes well posed. Figures $4 \mathrm{c}$ and $\mathrm{d}$ show the estimated $K_{s}$ and $\alpha$ values with the two fluxes; notice that the estimates are identical to the true values - the inverse problem is well posed. We emphasize that the other parameter $n$ in the VG model was assumed known a priori in this example.

If it is impossible to acquire the necessary information to uniquely identify the parameters of individual blocks, one may amend the problem by determining effective parameters for an equivalent homogeneous media. For instance, if only the specific discharge and sparse pressure head measurements along the heterogeneous column are available, seeking effective parameters such as $K_{s}$ and $\beta$ in the Gardner model, or $K_{s}, \alpha$, and $n$ of the VG model, is an alternative approach. However, to uniquely identify these effective parameters, conditions as discussed for steady-state flow through unsaturated homogeneous media must be met, except that mean pressure head distributions are used as demonstrated by Yeh (1989). Again, the effective hydraulic conductivity parameters so obtained are not the true hydraulic conductivity parameter values of each block but those hydraulic conductivity parameters for an equivalent homogeneous medium that will reproduce the specific discharge and yield the mean pressure distribution.

2.2.4. Transient unsaturated flow. For transient flow in an unsaturated heterogeneous medium, it is difficult to show in a simple manner conditions required to make an inverse problem well posed. However, the rule of thumb is that the initial and boundary conditions, and many sets of spatial steady state head distributions, and temporal head and moisture distributions during transient flows must be known. Inclusion of two or three fluxes is indispensable to make the inverse problem well posed. In other words, one must have adequate information to create a sufficient number of independent equations so that a unique solution is possible.

The previous examples assume that the size of each soil block is the same and known. If the size of each block varies and the permutation of the blocks is unknown, the problem becomes even more complex. Nevertheless, if the average block size and the maximum and minimum values of the hydraulic conductivity values are known, the inverse problem will become better posed. While our discussion focused on one-dimensional flow problems, the same principles are also applicable to multi-dimensional flow problems. In multi-dimensional flow problems, the hydraulic conductivity anisotropy of the equivalent homogeneous medium, or the anisotropy of each block, may have to be considered. This means that more parameters may need to be identified. Therefore, additional information about the system's spatial and temporal responses must be acquired.

\section{Methods For Solving The Well-Posed Inverse Problems.}

Two types of approaches have been used to solve inverse problems in subsurface hydrology: direct methods and indirect methods (see Neuman, 1973; Yeh, 1986). Direct methods are methods for determining primary variables directly using secondary information from the inversion of the governing flow equation. For example, the hydraulic conductivity distribution along the column in Figure 1 can be directly determined by using Darcy's law or solving the system of equations (12). Direct methods often encounter numerical instability 
problems. A small error in the head data may lead to erroneous solutions for the conductivity (e.g., negative values), even if the prerequisites are met. Consequently, direct methods are often avoided. The iterative approach employed in the successive linear estimator (see section 5.2), however, appears to have overcome this difficulty.

Indirect methods, in contrast to direct methods, are a trial and error approaches. That is, one continuously guesses and adjusts values of the unknown parameters or boundary/initial conditions until the simulated heads (or other information) agree with the observed ones, thus leading to a minimum output error (MOE) approach. This process is generally referred to as model calibration. Obviously, adjustment of parameter values can be carried out either manually or using some mathematical algorithm (e.g., gradient search techniques) to arrive at the best-fit parameter values using a least-squares criterion. Indirect methods generally avoid numerical instability problems associated with direct methods. Indirect methods may, however, lead to a local minimum and, in turn, an incorrect solution.

In spite of their limitations, both the direct and indirect methods, in theory, should produce the exact solution if the inverse problem were well posed. On the contrary, no unique solution will exist if the problem is ill posed in spite of having a very effective solution technique. The fact is that if many global minima exist, most or all solution techniques will yield large numbers of solutions to the inverse problem. However, if some prior information about the conductivity distribution (e.g., the average block size, the maximum and minimum conductivity values), in addition to sparse observed responses of a system, is given, the solution can be better constrained. Furthermore, as illustrated previously, if one focuses on estimating an effective hydraulic conductivity field that can reproduce the conditioned mean system response, the inverse problem becomes a well-posed problem. More importantly, the conditional effective parameters also provide a statistically meaningful solution to the forward problem: one can then predict the ensemble mean response of the system. Uncertainty associated with the prediction subsequently can be quantified statistically.

\section{Stochastic Conceptualization of Inverse Problems}

The discussions above suggest several points concerning inverse problems for heterogeneous media. First, in theory, the inverse problem of flow in variably saturated media can be well posed and parameters can be identifiable if information about the system response, fluxes, and boundary and initial conditions are fully specified. Applications of inversion theories to laboratory and field experiments, however, are always ill posed because necessary information is always fragmentary, corrupted with noise, or compromised by other complications or limitations. The ill-posed problem, however, can be improved, if for example, the average size of soil blocks, and the maximum and the minimum conductivity values as in Figure 1, are used to constrain the inverse solution. The inverse problem can even become well posed if one seeks conditional effective parameters that reproduce the conditional mean behavior of the medium, although the parameters are not the true solution.

Because of these considerations, a probabilistic description (or stochastic representation) of the hydraulic properties becomes most appropriate. That is to say, each of the properties of a geological formation should be considered as a stochastic process with an infinite number of possible realizations, characterized by a joint probability distribution. The first moment of the joint probability distribution (the mean) provides information about the most likely value of the 
property. The second moment (the spatial covariance function) specifies the variance and correlation structure of the process, analogous to a description of maximum and minimum values of the properties and the average block size, as in previous discussions, respectively. If one adapts the stochastic representation of the hydraulic property, the corresponding response of the formation to an excitation is then considered as a stochastic process, which also has an infinite number of possibilities (as in Figure 1).

With limited secondary information (i.e., information about the secondary variable or system response), an inverse model thus is best perceived as a means that can produce the property and response fields that agree with properties and responses at sample locations. In addition, these fields must satisfy the statistics (i.e., the mean and covariance) describing their spatial variability, while the governing equation must describe the underlying physical process. In a conditional probability concept, this resultant field is a conditional realization of the property or response field, among many possible realizations in the ensemble. While many possible realizations of such a conditional field exist (i.e., nonunique solutions), the conditional mean field is unique. This field also represents the most likely solution to the inverse problem, even though this may not necessarily be the true field of the soil profile or geological formation. Its deviation from the true fields is quantified through the conditional variance (uncertainty). As more pieces of primary and secondary information are acquired, the conditional mean will gradually resemble the true realization of the property field of the given geological formation, and the uncertainty progressively vanishes.

\section{Geostatistically Based Inverse Approaches.}

Geostatistically based inverse approaches to be discussed below evolved from the stochastic conceptualization of the inversion problem. In this section, we will elaborate on cokriging, a successive linear estimator, and a sequential conditioning approach, with applications to hydraulic tomography, electrical resistivity tomography, and finally to stochastic fusion of information.

\subsection{Cokriging}

Geostatistically based inverse techniques rely on the classical linear predictor theory that considers spatial correlation structures of flow processes (such as pressure heads and velocities) and hydraulic properties. They also take in account possible cross-correlation between the flow processes and the hydraulic properties of porous media. This approach has been widely used to estimate transmissivities, heads, velocities, and concentrations of pollutants in highly heterogeneous aquifers (e.g., Kitanidis and Vomvoris, 1983; Hoeksema and Kitanidis, 1984 and 1989; Rubin and Dagan, 1987; Gutjahr and Wilson, 1989; Sun and Yeh, 1992; Harvey and Gorelick, 1995; Yeh et al., 1995 and 1996). The approach has also been used to estimate water content distributions, based on combined measurements of water content, soil-water pressure head, soil surface temperature, and/or soil texture (e.g., Vauclin et al., 1983; Yates and Warrick, 1987; and Mulla, 1988).

The first inverse technique based on geostatistics (cokriging) was developed to identify unsaturated hydraulic parameters in heterogeneous vadose zones during steady-state uniform flow (Harter and Yeh, 1996) and non-uniform flow (Yeh and Zhang, 1996). Later, Hughson and 
Yeh (1998) extended the approach to transient unsaturated flow. A brief discussion of the principle of cokriging is given below (see Hughson and Yeh, 1998 for additional details).

To estimate the parameters $K_{s}, \alpha$, and $n$ in the VG model, the cokriging approach relies on measurements of the parameters, pressure heads, and moisture contents, and their statistical moments (i.e., mean, covariances and cross-covariances). The covariances and crosscovariances of the pressure head and the moisture content are calculated using a first-order analysis as described below. First, state variables are expanded in a Taylor series around the means in the general form

$$
u=\langle u\rangle+\left.\sum_{i} \chi_{i} \frac{\partial u}{\partial \mathrm{X}_{i}}\right|_{\left\langle\mathrm{X}_{i}\right\rangle,\langle u\rangle}
$$

where $u$ is the state variable $h$ or $\theta$, the summation is over the stochastic parameters, and terms of second and higher order are neglected. In this expression $\chi_{i}=X_{i^{-}}<X_{i}>$ represents the zero mean perturbation of a $\log$ transformed hydraulic parameter such as $f=\ln \left(K_{s}\right)-<\ln \left(K_{s}\right)>, a=\ln (\alpha)$ $<\ln (\alpha)>$, or $v=\ln (n)-<\ln (n)>$, while the angle brackets denote expectation. Perturbations in the state variables, $v=u-\langle u\rangle$, also have a mean of zero. Sensitivity derivatives of (16) are computed with an adjoint state method. Details of this derivation can be found in Sun and Yeh (1992), Li and Yeh (1998 and 1999), and other references cited by those authors.

Notice that one must derive the mean pressure head, $\langle h\rangle$, first in order to evaluate the sensitivities discussed above. To do so, the mean equation is assumed to be the same as the Richards equation (1), while $K(h)$ and $C(h)=d \theta / d h$ are assumed to be described by (3) and derived from (2), respectively, with parameter values set to their mean values (Yeh, 1998). Thus, a zero-order mean pressure head can be obtained by solving

$$
\left[\omega S_{s}+C(\langle h\rangle)\right] \frac{\partial\langle h\rangle}{\partial t}=\nabla \cdot[K(\langle h\rangle) \nabla(\langle h\rangle+z)]
$$

using a finite difference or finite element code, such as MMOC3, developed by Srivastava and Yeh [1992], HYDRUS-2D (Šimůnek et al., 1999), or other software.

Once the mean pressure head is obtained, the above sensitivity equations can be used to calculate covariances and cross-covariances. Given that the flow domain is discretized into $j$ blocks or elements, multiplying (16) by perturbations $f, a$, and $v$, and subsequently taking the expectation, results in expressions for the cross-covariances of the head and moisture contents with the hydraulic properties as follows

$$
\mathbf{R}_{v \chi}=\mathbf{R}_{\chi \chi} \mathbf{J}_{v \chi}
$$

In these expressions $\mathbf{R}_{u \chi}$ is notation for the cross-covariance matrix $\langle u \chi\rangle$ of zero mean perturbations in parameters and state. The $j \times j$ matrices $\mathbf{R}_{\chi \chi}$ for the covariance functions of the $\log$-transformed perturbations of the hydraulic properties $K_{s}, \alpha$, and $n$ are assumed known. Cross-covariance matrices $\mathbf{R}_{v \chi}$ have dimensions $j \times n_{d}$ where $n_{d}$ refers to the number of $h$ or $\theta$ data locations. The Jacobian (sensitivity) matrices $\mathbf{J}_{v \chi}$ also are $j \times n_{d}$. In the formulation of (18) we have assumed that the hydraulic properties are independent. Note that our assumption of independence represents the worst-case scenario in that information about one parameter tells us nothing about the others. 
Covariances of the perturbations in the pressure head, $\psi=h-\langle h\rangle$, and moisture content, $s=\theta-<\theta>$, are derived by multiplying (16) by the perturbations, taking the expectation, and substituting (18) as

$$
\mathbf{R}_{u w}=\sum_{\chi} \mathbf{J}_{v \chi}^{T} \mathbf{R}_{\chi \chi} \mathbf{J}_{w \chi}
$$

where $T$ indicates transpose. Covariances are obtained, where $v$ and $w$ are perturbations of the same state variable, and cross-covariances where $v$ is $h$ and $w$ is $s$. Covariances of the secondary information in (19) are $n_{d} \times n_{d}$ matrices. Note that the cross-covariance matrix computed in (19) need not be symmetric. Second-order approximations of these covariances can be obtained by a method developed by Liedl (1994).

Using the covariances, a first-order estimate of the perturbations in the log-transformed hydraulic properties can be obtained by solving the cokriging equations. The estimated perturbations will match data of the hydraulic property of interest, and incorporate secondary information from the pressure head and moisture content variables. The cokriging equations are the matrix equation

$$
\left[\begin{array}{lll}
\mathbf{C}_{\chi \chi} & \mathbf{C}_{s \chi} & \mathbf{C}_{\psi \chi} \\
\mathbf{C}_{s \chi}^{T} & \mathbf{R}_{s s} & \mathbf{R}_{\psi s} \\
\mathbf{C}_{\psi \chi}^{T} & \mathbf{R}_{\psi s}^{T} & \mathbf{R}_{\psi \psi}
\end{array}\right]\left[\begin{array}{c}
\lambda_{\chi} \\
\lambda_{s} \\
\lambda_{\psi}
\end{array}\right]=\left[\begin{array}{c}
\mathbf{R}_{\chi \chi}^{T} \\
\mathbf{R}_{s \chi}^{T} \\
\mathbf{R}_{\psi \chi}^{T}
\end{array}\right]
$$

where the symbol $\chi$ indicates the primary variable being estimated, and $\mathbf{C}_{\chi \chi}, \mathbf{C}_{\mathrm{x} \chi}$, and $\mathbf{C}_{\psi \chi}$ represent covariances and cross-covariances of the data locations, which are subsets of the covariance and cross-covariance matrices obtained from (18) and (19). Note that the top element on the right hand side of (20), $\mathbf{R}^{\mathrm{T}}{ }_{\chi \chi}$, is the specified covariance of the parameter field. It is a $j$ by number of parameter data subset of $\mathbf{R}_{\chi \chi}$. The $n_{d} \times j$ matrices $\lambda_{\chi}, \lambda_{s}$, and $\lambda_{\psi}$ are the cokriging weights applied to data of the primary variable, and secondary information on moisture content and pressure. On the right hand side of (20) are the covariances and cross-covariances of the data locations with the primary variable to be estimated. These transposed covariance matrices have rows equal to the number of data and $j$ columns. The cokriging weights are also matrices of the same dimension. Once the weights are evaluated, linear estimates of the hydraulic properties are

$$
\chi^{*}=\lambda_{\chi}^{T} \chi_{d}+\lambda_{s}^{T} s_{d}+\lambda_{\psi}^{T} \psi_{d}
$$

where $\chi^{*}$ is a $j \times 1$ vector of the estimated perturbation in a hydraulic property, and $\chi_{d}, s_{d}$, and $\psi_{d}$ are data of perturbations in the hydraulic property, moisture content, and pressure head. The conditional covariances (measures of uncertainty associated with the estimates) are evaluated using (22) to be discussed in a later section.

For a mildly heterogeneous aquifer, where the perturbation of the natural logarithm of the hydraulic conductivity is linearly related to the perturbation head field, it can be shown that cokriging and classical least squares optimization approaches yield identical solutions. This is true if the least squares optimization approach starts with kriged parameter estimates and considers the spatial covariance of the parameter (Kitanidis; 1986; Carrera and Glorioso, 1991) . However, cokriging differs from the least squares approach in many ways. The least squares approach is a regression model that considers only the sensitivity matrix (i.e., model cross- 
correlation structure) of the model response to change in the parameter. On the other hand, cokriging is a linear estimator based on observations. It uses the spatial covariance of the parameter and the cross-covariance of the parameter and the response of the model to yield the estimate that satisfies the minimum-mean-square-error criterion. Notice that differently to the sensitivity matrix, the cross-covariance is a product between the spatial covariance of the parameter and the sensitivity matrix. Furthermore, computational algorithms of the two approaches are quite different. Cokriging uses observations to estimate the parameter at one location from the other, while the classical least-squares approach simultaneously estimates the parameter at all locations. Therefore, the matrix in the least-squares approach is much larger than in cokriging (Kitanidis, 1997) in cases where the number of observations is smaller than the number of locations where parameters are to be estimated. More importantly, parallel computing can add to the power of cokriging because of its unique computational algorithm.

Of course, cokriging implicitly assumes that the stochastic parameter is a Gaussian process. Whether or not the process is Gaussian, the argument is that if the process is Gaussian then cokriging is optimal, whereas if the process is non-Gaussian, then cokriging is merely a best linear predictors.

Using cokriging, Yeh and Zhang (1996) showed that the unsaturated hydraulic parameters of a heterogeneous vadose zone could be identified if sufficient pressure and degree of saturation information was available. They found that, under wet conditions, measurements of the pressure improved estimates of the saturated hydraulic conductivity. Under dry conditions, measurements of saturation enhanced the estimate of the pore-size distribution parameter, $\beta$, in the Gardener model. This finding is consistent with previous rudimentary discussions of wellposedness of the inverse problem for unsaturated flow.

Li and Yeh (1999) applied cokriging to estimate the hydraulic conductivity, using pressure head, solute concentration, and solute arrival time measurements in a hypothetical, heterogeneous vadose zone subject to steady-state infiltration at different degrees of saturation. Their analysis showed that the performance of cokriging using either head or concentration measurements deteriorates as the medium becomes less saturated. They attributed this phenomenon to an increase in nonlinearity between head or concentration and the conductivity as the medium becomes less saturated, and the linear predictor nature of cokriging.

Among pressure head, solute concentration, and solute arrival time measurements, $\mathrm{Li}$ and Yeh (1999) found that pressure head measurements of steady state flow fields were the most useful secondary information for estimating the $K_{s}$ field using cokriging. They attributed this finding to several factors. First, the nonlinear relationship between head and $K_{s}$ may have been relatively mild in the cases studied. Additionally, the assumption of ergodicity was approximately satisfied for steady state flow. In other word, the ensemble mean head distribution evaluated with mean values of the parameters closely approximates the spatial mean head distribution. Such an existence of ergodicity reduces the variance of the head, and consequently improves the linearity between head and conductivity. Conversely, the ergodicity assumption cannot be easily satisfied for the solute transport case. Only when a solute plume has traveled over enough correlation lengths, has sampled enough heterogeneity, and has become a Fickian process, will the ergodicity assumption be satisfied. Because of lack of ergodicity, the variance of a concentration perturbation can be very large and the cokriging estimation using concentration measurements can be unsatisfactory. 
Note that the concentration of a tracer observed at a given location is a result of both convection and dispersion processes. Let us assume that convection is the dominant process and neglect dispersion. The convection process is a function of not only the hydraulic conductivity but also the hydraulic gradient and the moisture content. Cross correlation between the concentration and the hydraulic conductivity is thus not as strong as the relationship between pressure head and hydraulic conductivity. This suggests that for estimating the hydraulic conductivity, concentration measurements are in general somewhat less effective. The same argument applies also to arrival times of a tracer concentration. However, Li and Yeh (1999) found that estimates of the conductivity based on head measurements could be improved by incorporating additional concentration information.

\subsection{Successive Linear Estimator}

While cokriging is useful, it is only a linear predictor. The relation between primary and secondary variables of the vadose zone is highly nonlinear. Therefore, cokriging cannot fully exploit available secondary information. To overcome this limitation, Zhang and Yeh (1997) adapted a successive linear estimator technique (SLE, Yeh et al., 1996) to the vadose zone inverse problem.

The SLE consists of seven steps. Step (1) starts with a linear estimator (cokriging as discussed in the previous section) which uses primary and secondary information to estimate the value of the primary variable at $j$ locations where no information of the variable is available. In Step (2), the covariance of the primary variable (a measure of uncertainty of the estimate) is modified to reflect the effects of available information. That is,

$$
\mathbf{R}_{\chi \chi}^{(r+1)}=\mathbf{R}_{\chi \chi}^{(r)}-\check{\mathbf{R}}_{\chi \chi}^{(r)} \lambda_{\chi}-\mathbf{R}_{h \chi}^{(r)} \lambda_{h}-\mathbf{R}_{s \chi}^{(r)} \lambda_{s}
$$

where $\breve{\mathbf{R}}_{\chi \chi}^{(r)}$ is a $j \times n_{d}$ subset of $\mathbf{R}_{\chi \chi}^{(r)}$, covariance of $\chi$. The superscript in parentheses is the iteration index and $r=0$ at this time. In Step (3), the newly estimated variable field from (1) is used to simulate the process field, $h$ and $s$, using a forward simulation model for the physical process. In Step (4), the conditional covariances, $\mathbf{R}_{h h}^{(r+1)}$ and $\mathbf{R}_{s s}^{(r+1)}$ and their cross-covariances, $\mathbf{R}_{h \chi}^{(r+1)}$ an*d $\mathbf{R}_{s \chi}^{(r+1)}$ are updated using a first-order analysis. For Step (5), these newly evaluated covariances and cross-covariances compute new weights, $\lambda$ :

$$
\left[\begin{array}{ll}
\mathbf{R}_{h h}^{(r)} & \mathbf{R}_{h s}^{(r)} \\
\mathbf{R}_{h s}^{(r)} & \mathbf{R}_{s s}^{(r)}
\end{array}\right]\left[\begin{array}{c}
\lambda_{h}^{(r)} \\
\lambda_{s}^{(r)}
\end{array}\right]=\left[\begin{array}{l}
\left(\mathbf{R}_{h \chi}^{(r)}\right)^{T} \\
\left(\mathbf{R}_{s \chi}^{(r)}\right)^{T}
\end{array}\right]
$$

Step (6) is where the new weights, along with the difference between simulated $h^{(r)}$ and $s^{(r)}$ and observed $h_{d}$ and $s_{d}$, are used to improve the estimate of the primary variable:

$$
\hat{\chi}^{(r+1)}=\hat{\chi}^{(r)}+\lambda_{h}^{(r)}\left(h_{d}-h^{(r)}\right)+\lambda_{s}^{(r)}\left(s_{d}-s^{(r)}\right)
$$

where $\hat{\chi}^{(r)}$ represents the primary variable estimate at iteration $r$. In Step (7), the weights are employed to update conditional covariances for the next iteration.

$$
\mathbf{R}_{\chi \chi}^{(r+1)}=\mathbf{R}_{\chi \chi}^{(r)}-\mathbf{R}_{h \chi}^{(r)} \lambda_{h}-\mathbf{R}_{s \chi}^{(r)} \lambda_{s}
$$

This newly updated primary variable field (4) and new conditional covariances (25) are

employed again in Steps (3) and (4), followed by Steps (5) through (7). In essence, Steps (3) 
through (7) are repeated until no improvement in the estimate of the primary variable is found (i.e., the solution converges).

Similar to a nonlinear maximum a posteriori (MAP) method (see McLaughlin and Townley, 1996), the SLE is also based on a Bayesian framework but differs from MAP in many aspects (Kitanidi, 1997). More importantly, during nonlinear iterations, our SLE updates the second moment of the posteriori probability of the primary variable in a consistent manner using a first-order approximation approach. For a well-posed inverse problem (saturated or unsaturated flow), the SLE converges to the true solution (as shown in Figure 4). In addition, the SLE accounts for measurement error in the estimation.

Zhang and Yeh (1997) showed that the SLE approach could yield more detailed images of hydraulic parameters than cokriging for identifying vadose zones parameters. SLE can more clearly depict high- and low-permeability zones that can significantly affect predictions of contaminant transport in the vadose zone.

Zhang and Yeh (1997) further refined the geostatistical inverse approach. While both MOE and cokriging are regression-type models, the cokriging approach, in contrast to the classical MOE, uses a prior correlation structure to define the generic heterogeneity pattern of the primary variable at a given field site. A few measurements of the primary variable tailor this pattern to yield a site-specific heterogeneity map. This map can then be improved using available secondary information, depending upon the cross-correlation between primary and secondary information and the model describing their relation. The SLE approach considers the nonlinear relation between the primary variable and secondary information, and can thus reveal a more detailed image of heterogeneity.

Based on the SLE principle, Hughson and Yeh (2000) developed an inverse model for three-dimensional, transient flow in heterogeneous vadose zone. In addition, a technique was developed to allow pressure head and water content data, obtained at different times in the inverse modeling effort, to be sequentially included in the inversion. This technique eliminates numerical difficulties associated with simultaneous inclusion of a large number of data sets. The method increases the efficiency of the model such that inverse modeling of a three-dimensional, heterogeneous vadose zone with a large number of primary variables becomes possible. Using this tool, they investigated the efficacy of estimating VG parameters using pressure and moisture content measurements at relatively early, intermediate, and late time periods. For the cases investigated, they concluded that late time data provided the best estimates. While their results were reasonable, imposing different flow rates to establish different steady flow regimes could have made the problem better posed and thus enhanced their estimates (see section 2.2.3.).

In spite of the development of a three-dimensional inverse model, accurately identification of a large number of primary variables in a three-dimensional vadose zone demands a large amount of secondary information. Traditional means to collect the secondary information often required installation of numerous soil sampling sites and/or boreholes. Such excessive and invasive sampling is impractical and undesirable. The collection of copious secondary information with a minimum number of boreholes becomes then an important research objective. The following sections examine various approaches to overcome this issue.

\subsection{Hydraulic/Pneumatic Tomography.}


Hydraulic or pneumatic tomography tests are one possible approach to acquire a vast amount of secondary information without resorting to extensive, expensive invasive sampling. Hydraulic/pneumatic tomography can generate many pressure measurements with a fixed number of boreholes (Gottlieb and Dietrich, 1995; Butler et al., 1999; Yeh and Liu, 2000; Liu et al., 2002; Vesselinov et al., 2000). For example, using packers, two fully screened wells in an aquifer can be partitioned into many intervals. By sequentially pumping water at different intervals, and monitoring the steady-state head response at the other wells and packed-off intervals, many pairs of head/discharge data sets can be obtained with only two wells. This vast amount of information may yield additional independent equations for the inverse process as discussed in 2.1. Thus, it allows further conditioning of the inverse solution as illustrated in section 2.2.1 through 2.2.4. While the above example deals mostly with saturated flow, a tomographic survey concepts can be equally well applied to, for example, unsaturated infiltration tests.

Interpretation of the vast amount of data sets acquired from tomographic tests demands efficient inverse algorithms. The discussion in section 5.2 describes the SLE method for evaluating the secondary information collected during one excitation (e.g., an injection test) in a tomographic experiment. The method can simultaneously include all secondary information collected during all excitations in a tomographic test. However, the system of equations, (20) and (23), can become extremely large and ill conditioned, which may make it difficult to obtain stable solutions to the equations (Hughson and Yeh, 2000). To avoid this problem, secondary information collected from excitations at different locations could be used sequentially. Specifically, our sequential method will start the inversion with secondary information collected from the excitation at one selected location. Once the estimated field converges to given criteria, the newly estimated conditional mean primary field and its conditional covariance are used as prior information for the inversion of the next excitation. That is, the conditional mean of $\chi$ is used to evaluate the conditional means of $h$ and $s$, and sensitivity matrices associated with the excitation at the new location. Using the first-order analysis, the sensitivity matrix and the conditional covariance, $\chi$, yield the $h$ and $s$ covariances and their cross-covariances with $\chi$, which are subsequently employed to derive the new weights. With the conditional mean $h$ and $s$, new weights, and the and observed $h_{d}$ and $s_{d}$, equation (24) then produces a new estimate of $\chi$, representing the estimate based on information from the excitation at the new location. An iterative process (similar to Steps (3) through (7) in section 5.2) is then employed to include the nonlinear relationship between $h$ and $\chi$. Once the solution converges and the iterative process stops, the same procedure is applied to information from the next excitation. This sequential process continues until the data sets created from all excitations are used in the inversion.

The above sequential approach allows accumulation of high-density secondary information obtained from a tomographic survey, while maintaining the system of equations at a manageable size that can be solved with the least numerical difficulties.

Using this sequential SLE approach, Yeh and Liu (2000) and Liu et al., (2002) showed that the tomographic aquifer test has significant advantages over traditional pumping tests. It can provide a detailed image of three-dimensional aquifer heterogeneity with the same number of wells used in a traditional pumping test. The tomographic test is also superior to any inverse method that uses only one set of secondary information created by one excitation to the aquifer. The approach generates a large database of information about the behavior of the aquifer in 
response to excitations at different locations so as to constrain possible solutions to an inherently ill-posed inverse problem.

Pumping or injection tests using air in the unsaturated zone are one of many possible approaches to determine the permeability and porosity of unsaturated soil and fractured rocks. In principle, air injection tests are very similar to their hydraulic counterparts conducted in fully water-saturated media. Air is either injected or withdrawn from sections of boreholes isolated by means of inflatable packers, with the pressure responses monitored in observation wells and packed-off intervals. The pressure response in observation wells can be related to pneumatic flow parameters such as permeability and porosity through analytical techniques or numerical inverse modeling. Pneumatic tomography (i.e., cross-hole pneumatic injection tests conducted sequentially, and its corresponding inverse interpretation) has recently been proposed as a method for characterizing subsurface heterogeneity (Vesselinov et al., 2000). In general, experience with pneumatic injection and gaseous tracer experiments in fractured rocks is limited. Much of this experience has been gained recently during pneumatic injection tests in tuffs at Yucca Mountain, Nevada (LeCain and Walker, 1994; LeCain, 1996, 1998; Wang et al., 1998; Huang et al., 1999), in Box Canyon, Idaho (Benito et al., 1998, 1999), and at the Apache Leap Research Site (ALRS) near Superior, Arizona (Trautz 1984; Yeh et al., 1988; Rasmussen et al., 1990, 1993; Guzman et al., 1996; Illman et al., 1998).

In spite of their ability to create many data sets to constrain the inverse solution, hydraulic and pneumatic tomography also have their limitations. The effectiveness of tomographic tests has been found to decrease rapidly, indicating that excessive sequential excitation often produces only redundant information (Yeh and Liu, 2000) because secondary information is always collected at the same locations. In addition, data are often affected heavily by barometric pressure fluctuations (Illman et al., 1998; Illman and Neuman, 2001), which causes the effectiveness of the data to decline rapidly. Accordingly, we believe that acquisition of high-density and accurate secondary information throughout the vadose zone remains the only viable means to enhance the ability of an inverse model to produce high-resolution subsurface images.

\subsection{Electrical Resistivity Tomography For Monitoring the Vadose Zone}

Although geophysical surveys cannot provide the primary information needed for hydrologic modeling, they can be extremely cost-effective, indirect tools for monitoring hydrologic processes, and for detecting geological structures. Recently, the electrical resistivity tomography (ERT) surveys have been demonstrated to be a viable technology for collecting high-density secondary vadose zone information without excessive invasive samplings (Binley et al., 2001. Daily et al., 1992). Similar to hydraulic tomography, ERT emits DC currents at a point in space and monitors the electrical potential at other locations. By moving the DC source location, one can generate many electrical potential fields and source pairs, from which a 3-D image of the change in resistivity can be derived using an inverse model. Assuming the change in resistivity mimics the change in moisture content, water flow in the vadose zone can be monitored. Furthermore, assuming a universal moisture content and resistivity relation (i.e., Archie's law), a three-dimensional image of the moisture content distribution over a large volume of geological material can thus be obtained (Daily et al., 1992). Electrical resistivity 
tomography appears to offer great promise as a tool for assembling the extreme data requirements needed for accurate hydrologic inversion modeling.

ERT, unfortunately, relies on inversion of the potential equation. With limited electrical potential measurements, the ERT inversion problem become ill posed. The estimated resistivity field thus may be subject to great uncertainty. Nevertheless, it is generally agreed that ERT can detect the general pattern of change in resistivity and thus, the pattern of change in moisture content. Furthermore, the resolution of the ERT image can be easily improved by deploying a large number of sensors within boreholes and at the soil surface. The relative inexpensiveness of the sensor makes such a deployment feasible, thus making ERT a highly desirable monitor tool for vadose zone investigations. Nonetheless, a recent field study (Baker, 2001 and Yeh et al., 2002) indicated that the parameters of Arche's law (i.e., the resistivity/moisture content relation) exhibit profound spatial variability. This variability compounds the difficulty in translating resistivity to moisture content. In other words, at some location, a small change in resistivity may reflect a large change in water content, while at other locations a substantial change in the resistivity may correspond to only a small change in the water content. Consequently, the ERT's ability to yield an accurate image of change in moisture content, or more critically the moisture content itself, remains to be proven.

\section{Stochastic Fusion of Information}

Several aspects about the characterization and monitoring of vadose zones arise from our previous discussions. 1) Geologic materials exhibit random spatial variations in physical properties (i.e., hydraulic conductivity, porosity, and electrical resistivity) on a multiplicity of scales. Small-scale direct sampling of the entire vadose zone is in most or all cases virtually impossible. 2) Reliable inversions of variably saturated flow problems require a large amount of secondary information at high densities over the entire volume of the investigation, although many constraints prohibit the collection of such information. 3) Tomographic tests can produce additional data sets to further constrain the inversion but they have their limitations. 4) Geophysical surveys have the potential to collect a vast amount of information pertinent to hydrological inversion. However, significant spatial variation in the relationships between various hydrologic and geophysical properties diminishes the value of the information for its intended applications.

Because of these problems, assimilation of different types of information using a stochastic approach appears to be the only viable solution for characterizing and monitoring the vadose zone. In other words, while geological characterization, point measurements of hydrologic and geophysical properties, hydraulic tomography, and ERT surveys have their pros and cons, an integration of their individual strengths may facilitate a better mean for vadose zone monitoring and characterization. Both hydrological and geophysical inversion methods need to include all available secondary information (e.g., hydrologic, geological, and geophysical data) to better condition the ensemble of their primary variables. In addition, the integration must recognize that hydrologic information can provide useful constraints for the ERT inversion while, and on the other hand, the ERT inversion can furnish a vast amount of water content data for hydrological inverse modeling. Such a reciprocal nature of hydrologic and geophysical information and inversions thus demands a joint inversion that requires an iterative manner to fully utilize all available hydrological and geophysical data. Consequently, the conditional 
means, the best and unique estimate with the minimal uncertainty in a statistical sense, can be obtained, whereas the conditional variances then quantify their uncertainty. This is at the heart of the stochastic fusion of information concept to be described below.

The stochastic fusion approach comprises two major levels. At level 1 , the aim is to include all information or data sets related to ERT surveys to enhance their interpretations of images of spatial moisture distributions in the vadose zone. We believe that the sequential SLE algorithm presented in section 5.3 is most suitable for this purpose because of its linear estimator nature. The information may include our prior knowledge of resistivity correlation structure, voltages induced by the survey, point measurements of resistivity, resistivity-moisture relations, moisture retention curves, pressure heads collected from tensiometers, and moisture contents from neutron probes or time domain reflectrometers. Three-dimensional resistivity fields and moisture distributions in the vadose zone derived from the ERT inversion thus are consistent with hydrological and geophysical point measurements (see Liu, 2001). In short, Level 1 assimilates available primary and secondary information of the ERT process to derive the most likely estimate of resistivity field and its uncertainty conditioned on available hydrological and geophysical information. This is then translated to the most likely moisture content field and the uncertainty associated with that field. The same principle can be applicable to unsaturated hydraulic tomography.

Level 2 fusion intends to assimilate secondary information of the electrical current flow process created by ERT surveys, and the flow process induced by unsaturated hydraulic tomography. It will take an iterative approach to arrive at the best estimate of the primary variable fields and uncertainty. The flow chart shown in Figure 5 depicts a general concept of the fusion process. Specifically, during an unsaturated hydraulic tomography experiment, several water infiltration tests at different locations (packed-off intervals) could be conducted sequentially. During each test, some point measurements of the pressure head and moisture content are taken, while ERT is deployed to monitor movement of water. The resistivity image from ERT is improved by using Level 1 fusion technology to integrate hydrologic and geophysical information into the ERT inversion process. Thus, ERT is able to estimate not only changes in resistivity and water content, but also provides moisture content values and their conditional moments.

Estimates of the moisture content distributions, together with their conditional moments, are subsequently used as input to the unsaturated hydraulic inversion model (Hughson and Yeh, 2000) for the vadose zone to estimate three-dimensional distributions of the unsaturated hydraulic conductivity and moisture retention curves. In addition, conditional moments for the parameters can be derived. With the additional information about moisture content at locations where no samples were available, the hydrological inversion of unsaturated media properties thus improves, and a more detailed image of the hydraulic heterogeneity emerges. Simulations using the newly derived heterogeneity image then produce better moisture estimates and in turn resistivity values at locations where no measurements are available. The improved simulations of moisture contents/resistivity fields are next returned to the ERT inversion to improve the estimates of resistivity and, in turn, moisture content distributions. The iterative process continues until no further improvement in both hydraulic heterogeneity and moisture content estimates can be gained. This iterative procedure is repeated for each unsaturated hydraulic tomographic test. With such an iterative fusion of information, ERT becomes not only a better 
monitoring tool for moisture contents, but also an ideal tool for characterizing the unsaturated properties of the vadose zone.

Stochastic fusion of information also facilitates a better network design. The proposed technology can first be applied to small-scale field experiments. Uncertainty derived from the stochastic fusion algorithm for such small-scale experiments will then guide further sampling plans that will yield new information which is guided back to the algorithm to zoom into the existing scale of investigation, or expand the experiments to a larger domain.

We believe that the stochastic fusion technology is merely a common sense, logical approach that fine-tunes an initially coarse-scale approach to an increasingly fine-scale analyses. It uses geostatistical and geological information to prescribe the generic heterogeneity pattern of a field site. Point measurements of primary information then tailor the generic pattern to a sitespecific heterogeneity image. The secondary information further enhances the image of the primary variable, depending on the cross-correlation between the secondary information and the primary variable. When one type of secondary information is not sufficient to narrow the possible images of the primary variable distribution, it is likely that different types of secondary information can be employed alternatively or additionally. The iterative approach checks consistency of different types of information to reduce the effects of noise, to maximize the utility of the information, and to integrate information acquired at different scales. It derives the best solution to an ill-posed problem, in a statistical sense, and quantifies uncertainty associated with the estimate. Because the approach employs the sequential SLE algorithm, the technology further allows the sequential inclusion of data to reduce the cost of simultaneous employment of a large number of monitoring instruments, and to reduce the requirement of super computers. In essence, the approach transforms our common sense to a technology that fuses different types of data in a quantitative and cost-effective manner to characterize, monitor, and forecast processes in the vadose zone. Ultimately, the technology facilitates better decision-making.

To demonstrate the promise of the stochastic fusion technology, some preliminary results of our ongoing research project supported by DOE/EPA during the past few years are presented below. As part of this project we developed a methodology that fuses information obtained from ERT surveys, point hydrological measurements, and unsaturated hydraulic inversion modeling to better characterize and monitor the vadose zone. Figure 6a depicts the VG pore-size distribution $(\alpha)$ field for a synthetic vadose zone. The other VG parameters are assumed similarly to be heterogeneous (not shown here). A $\theta$ distribution corresponding to a steady infiltration from the center top portion of the vadose zone is shown in Figure $6 \mathrm{~b}$. These two figures are the basis of the following discussions.

We examine two cases in which the $\alpha$ field will be estimated using $\theta$ measurements, and the other parameter fields are assumed known beforehand. In Case 1, moisture content measurements are assumed available at six locations (black circles in Figure 6b). Using a theoretical moisture content covariance, the six measurements, and a kriging technique, an estimated $\theta$ distribution (Figure 7a) was derived. From this kriged $\theta$ distribution, $34 \theta$ values were then sampled at locations indicated by red circles. Afterward, our hydraulic inversion model was employed using these $\theta$ measurements in conjunction with one measurement of $\alpha$ (red circle in Figure 7a) to estimate the $\alpha$ distribution over the entire vadose zone. With this 
estimated $\alpha$ field, a forward simulation was carried out to yield a new $\theta$ distribution corresponding to the steady infiltration (plots of Figure 7c).

In Case 2, an ERT survey was conducted over the synthetic vadose zone with the true $\theta$ distribution (Figure 6b). An ERT inversion was then carried out in which the six $\theta$ measurements were used to constrain the inversion. Figure $7 \mathrm{~d}$ shows the estimated $\theta$ distribution from this inversion over the entire vadose zone. In comparison with Figure $6 \mathrm{~b}$, Figure $7 \mathrm{~d}$ reveals a striking similarity with the true $\theta$ field, indicating the advantage of fusing point measurements of $\theta$ and an ERT survey. Similar to Case 1, 34 moisture contents were sampled from the estimated $\theta$ field. Combining these samples with the six directly measured $\theta$ values and one $\alpha$ sample, our hydraulic inversion model produced an estimated $\alpha$ field for the entire vadose zone (Figure 7e). As expected, the estimated $\alpha$ field showed better agreement with the true $\alpha$ field (Figure 6a) than the one obtained from Case 1. This is attributed to the fact that more accurate $\theta$ data were obtained from this inversion than in Case 1 due fusion of information. Figure $7 \mathrm{f}$ shows plots of the simulated $\theta$ distribution from the estimated $\alpha$ field; the plots are only slightly better than Figure $7 \mathrm{~d}$ because of the greater effect of the other known parameters. Nonetheless, the hydraulic inversion would have yielded an even better estimate of the $\alpha$ field and, in turn, a better simulation of the moisture content distribution than Figure $7 \mathrm{f}$, if more $\theta$ information from the ERT survey (Figure 7d) had been used. 


\section{Conclusion}

The relatively simple examples presented in this paper illustrate necessary conditions for a well-posed inverse problem for steady-state and transient flow through homogeneous and heterogeneous porous media, as well as saturated and unsaturated conditions. The rule of thumb is that one must have sufficient information (including the specification of the flux boundary condition) to create enough independent equations. Thus, the problem is well posed and a unique solution is possible.

Specifically, for steady-state saturated flow problems, a detailed pressure head distribution and the flux must be specified to uniquely determine $K_{s}$. In the case of transient saturated flow, non-zero fluxes and the pressure head distributions at three different times are the required information to ensure uniqueness of the inverse solution for $K_{s}$ and $S_{s}$. Similarly, for unsaturated steady flow problems, knowledge of the pressure head distribution with at least one steady flux is needed to ensure a unique solution. For transient unsaturated flow, in addition to knowledge of the pressure head distribution at different times, information about the moisture content will enhance the inverse solution, whereas the flux again needs to be known. The rule of thumb holds for homogeneous as well as heterogeneous media. We hope that our discussion will shed light on the design for laboratory and field experiments, and facilitate better solution to the inverse problem.

With limited information, additional head and hydraulic conductivity data, and knowledge of average size of heterogeneities, and the maximum, and minimum values of the hydraulic conductivity can reduce ranges of possible head and conductivity distributions. Adapting the approach of conditional effective parameters, an ill-posed inversion problem with limited information can be transformed to a well-posed problem. Solutions to the inverse problem then yield effective parameters that predict mean response of a porous medium conditioned on available information.

Under field conditions, stochastic conceptualization of the inverse problem is inevitable. Geostatistically based inverse procedures appear promising because they utilize prior statistical information of the geological structure, point observations, and flow processes to yield conditional effective hydraulic parameters and their uncertainty.

To further reduce uncertainty of the conditional effective hydraulic parameters, and to make the parameters more representative of reality, assimilating a broad range of hydrologic, geologic, and geophysical information in the inversion becomes essential. We believe that the stochastic fusion of information approach offers much promise to accomplishing this task.

\section{Acknowledgements}

The first author is funded in part by a DOE EMSP96 grant through Sandia National Laboratories (contract AV-0655\#1) and a DOE EMSP99 grant through the University of Wisconsin, A019493, and in part by an EPA grant R-827114-01-0. This work does not necessarily reflect the views of DOE and EPA, and no official endorsement should be inferred. 


\section{References}

Baker, K., Investigation of direct and indirect hydraulic property laboratory characterization methods for heterogeneous alluvial deposits: Application to the Sandia-Tech vadose zone infiltration test site, Master thesis, Geosciences, New Mexico Tech, Socorro, NM, 2001.

Benito, P. H., P. Cook, B. Faybishenko, B. Freifeld, and C. Doughty, Box Canyon pneumatic connectivity study: Preliminary data analysis, Report LBNL-42359, Lawrence Berkeley National Laboratory, Berkeley, CA, 1998.

Benito, P. H., P. Cook, B. Faybishenko, B. Freifeld, and C. Doughty, Crosswell air-injection packer tests for the assessment of pneumatic connectivity in fractured, unsaturated basalt, Rock Mechanics for Industry, Amadei, Kranz, Scott and Smeallie (eds.), 843-850, 1999.

Binley A., P. Winship, R. Middleton, M. Pakar, and J. West, High-resolution characterization of vadose zone dynamics using cross-borehole radar, Water Resour. Res., 37(11), 2639-2652, 2001.

Butler, J.J., Jr., C.D. McElwee and G.C. Bohling, Pumping tests in networks of multilevel sampling wells: methodology and implications for hydraulic tomography, Water Resour. Res., 35(11), 3553-3560, 1999.

Carrera, J. and L. Glorioso, On geostatistical formulations of the groundwater flow inverse problem, Adv. Water Resour., 14(5), 273-283, 1991.

Daily, W., A. Ramirez, D. J. LaBrecque, and J. Nitao, Electrical resistivity tomography of vadose water movement, Water Resour. Res., 28(5), 1429-1442, 1992.

Gardner, W. R., Some steady state solutions of the unsaturated moisture flow equation with application to evaporation from a water table, Soil Sci. 85, 228-232, 1958.

Gardner, W. R., and F. J. Miklich, Unsaturated conductivity and diffusivity measurements by a constant flux method. Soil Sci. 93:271-274, 1962.

Gottlieb, J., and P. Dietrich, Identification of the permeability distribution in soil by hydraulic tomography, Inverse Problems, 11, 353-360, 1995.

Gutjahr, A. L., and J. L. Wilson, Cokriging for stochastic models, Transp. Porous Media, 4(6), 585-598, 1989.

Guzman, A. G., A.M. Geddis, M.J. Henrich, C.F. Lohrstorfer, and S.P. Neuman, Summary of Air Permeability Data From Single-Hole Injection Tests in Unsaturated Fractured Tuffs at the Apache Leap Research Site: Results of Steady-State Test Interpretation, NUREG/CR-6360, U.S. Nuclear Regulatory Commission, Washington, D.C., 1996. 
Harvey, C. and S. M. Gorelick, Mapping hydraulic conductivity: Sequential conditioning with measurements of solute arrival time, hydraulic head, and local conductivity, Water Resour. Res., 31(7), 1615-1626, 1995.

Harter, Th., and T.-C.J. Yeh, Conditional stochastic analysis of solute transport in heterogeneous, variably saturated soils, Water Resour. Res., 32(6), 1597-1610, 1996.

Hillel, D., V.D. Krentos, and Y. Stylianou, Procedure and test of an internal drainage method for measuring soil hydraulic characteristics in situ. Soil Sci. 114:395-400, 1972.

Hoeksema, R. J., and P. K. Kitanidis, An application of the geostatistical approach to the inverse problem in two-dimensional groundwater modeling, Water Resour. Res., 20(7), 1003-1020, 1984.

Hoeksema, R. J., and P. K. Kitanidis, Prediction of transmissivities, heads, and seepage velocities using mathematical modeling and geostatistics, Adv. Water Resour., 12, 90-101, 1989.

Huang, K., Y. W. Tsang, and G. S. Bodvarsson, Simultaneous inversion of air-injection tests in fractured unsaturated tuff at Yucca Mountain, Water Resour. Res., 35(8), 2375-2386, 1999.

Hudson, D. B., Wierenga, P. J. and Hills, R. G., Unsaturated hydraulic properties from upward flow into soil cores. Soil Sci. Soc. Am. J., 60, 388-396, 1996.

Hughson, D.L. and T.-C. J. Yeh, A geostatistically based inverse model for three-dimensional variably saturated flow, Stochastic Hydrology and Hydraulics, 12, 285-298, 1998.

Hughson, D. L., and T.-C. J. Yeh, An inverse model for three-dimensional flow in variably saturated porous media, Water Resour. Res., 36(4), 829-839, 2000.

Illman, W.A. and S.P. Neuman, Type-curve interpretation of a cross-hole pneumatic injection test in unsaturated fractured tuff, Water Resources Research: 37(3), 583-603, 2001.

Illman, W.A., D.L. Thompson, V.V. Vesselinov, and S.P. Neuman, Single-Hole and Cross-Hole Pneumatic Tests in Unsaturated Fractured Tuffs at the Apache Leap Research Site: Phenomenology, Spatial Variability, Connectivity and Scale, Rep. NUREG/CR-5559, U.S. Nuclear Regulatory Commission, Washington, D.C., September, 1998.

Kitanidis, P. K., and E. G. Vomvoris, A geostatistical approach to the inverse problem in groundwater modeling and one-dimensional simulations, Water Resour. Res., 19(3), 677-690,1983 .

Kitanidis, P. K., Parameter uncertainty in estimation of spatial functions: Bayesian analysis, Water Resour. Res., 22(4), 499-507, 1986. 
Kitanidis, P. K., Comment on "A reassesment of the groundwater inverse problem" by D. McLaughlin and L. R. Townley, Water Resour. Res., 33(9), 2199-2202, 1997.

Kool, J. B., J.C. Parker, and M.Th. van Genuchten, Determining soil hydraulic properties for onestep outflow experiments by parameter estimation. I. Theory and numerical studies, Soil Sci. Soc. Amer. J. 49:1348-1354, 1985.

LeCain, G. D. and J. N. Walker, Results of air-permeability testing in a vertical borehole at Yucca Mountain, Nevada, Proceedings of the Fifth Annual International Conference on HighLevel Radioactive Waste Management, May 22-26, Las Vegas, NV, 2782-2788, 1994.

LeCain, G. D., Air-injection testing in vertical boreholes in welded and nonwelded tuff, Yucca Mountain, Nevada, U.S. Geological Survey Water-Resources Investigations Report 96-4262, 1996.

LeCain, G. D., Results from Air-Injection and Tracer Testing in the Upper Tiva Canyon, Bow Ridge Fault, and Upper Paintbrush Contact Alcoves of the Exploratory Studies Facility, August 1994 through July 1996, Yucca Mountain, Nevada, Water-Resources Investigations Report 984058, 1998.

Li, B., and T.-C. J. Yeh,Sensitivity and moment analysis of head in variably saturated regimes. Water Resour. Res., 21(6), 477-485, 1998.

Li, B., and T.-C. J. Yeh, Cokriging estimation of the conductivity field under variably saturated flow conditions, Water Resour. Res., 35(12), 3663-3674, 1999.

Liedl, R., A conceptual perturbation model of water movement in stochastically heterogeneous soils, Adv. Water Res., 17, 171-179, 1994.

Liu, S., T. -C. J. Yeh and R. Gardiner, Effectiveness of Hydraulic Tomography: Numerical and Sandbox Experiments, Water Resources Research, in press, 2002.

Liu, S., A sequential inverse approach for hydraulic tomography and electrical resistivity tomography: an effective method for site characterization, pp185, Ph.D. dissertation, The University of Arizona, Tucson, 2001.

McLaughin, D. and L. R. Townley, A reassessment of the groundwater inverse problem, Water Resour. Res., 32(5), 1996.

Mulla, D. J., Estimating spatial patterns in water content, matric suction, and hydraulic conductivity, Soil Sci. Soc. Am. J., 52, 1547-1553, 1988. 
Neuman, S. P., Calibration of distributed parameter groundwater flow models viewed as a multiple-objective decision process under uncertainty, Water Resour. Res., 9(4), 1006-1021, 1973.

National Research Council, Rock Fractures and Fluid Flow: Contemporary Understanding and Applications, National Academy Press, Washington, D.C., 1996.

Rasmussen, T. C., D. D. Evans, P. J. Sheets, and J. H. Blanford, Unsaturated Fractured Rock Characterization Methods and Data Sets at the Apache Leap Tuff Site, NUREG/CR-5596, U. S. Nuclear Regulatory Commission, Washington D.C., 1990.

Rasmussen, T. C., D. D. Evans, P. J. Sheets, and J. H. Blanford, Permeability of Apache leap tuff: Borehole and core measurements using water and air, Water Resources Research, 29(7), 1997-2006, 1993.

Rose, C. W., W. R, Stern, and J. E. Drummond, Determination of hydraulic conductivity as a function of depth and water content for soil in situ. Aust. J. Soil. Res. 3, 1-9, 1965.

Rubin, Y., and G. Dagan, Stochastic identification of transmissivity and effective recharge in steady groundwater flow, 1, Theory, Water Resour. Res., 23(7), 1185-1192, 1987.

Šimůnek, J., and M. Th. van Genuchten, Estimating unsaturated soil hydraulic properties from multiple tension disc infiltrometer data, Soil Science, 162(6), 383-398, 1997.

Šimůnek, J., O. Wendroth, and M. Th. van Genuchten, A parameter estimation analysis of the evaporation method for determining soil hydraulic properties. Soil Sci. Soc. Am. J. 62:894-905, 1998.

Šimůnek, J., M. Šejna, and M. Th. van Genuchten, The HYDRUS-2D software package for simulating two-dimensional movement of water, heat, and multiple solutes in variably saturated media. Version 2.0, IGWMC - TPS - 53, International Ground Water Modeling Center, Colorado School of Mines, Golden, Colorado, 251pp., 1999.

Šimůnek, J., and M. Th. van Genuchten, Using the HYDRUS-1D and HYDRUS-2D codes for estimating unsaturated soil hydraulic and solute transport parameters, in van Genuchten, M. Th., F. J. Leij, and L. Wu (eds.) Characterization and Measurement of the Hydraulic Properties of Unsaturated Porous Media, University of California, Riverside, CA, 1523-1536, 1999.

Srivastava R., T.-C.J. Yeh, A three dimensional numerical model for water flow and transport of chemically reactive solute through porous media under variably saturated conditions. Water Resour. Res., 15(6), 275-287, 1992.

Sun, N.-Z., and W. W.-G. Yeh, A stochastic inverse solution for transient groundwater flow: parameter identification and reliability analysis, Water Resour. Res., 28(12), 3269-3280, 1992. 
Toorman, A.F., P.J. Wierenga, and R.G. Hills, Parameter estimation of soil hydraulic properties from one-step outflow data. Water Resour. Res. 28:3021-3028, 1992.

Trautz, R. C., Rock Fracture Aperture and Gas Conductivity Measurements In-Situ, M. S. thesis, Dep. of Hydrol. and Water Resour., Univ. of Ariz., Tucson, 1984.

van Dam, J.C., J.N.M. Stricker, and P. Droogers, Inverse method for determining soil hydraulic functions from multi-step outflow experiments. Soil Sci. Soc. Amer. J. 58:647-652, 1994.

van Genuchten, M. Th., A closed-form equation for predicting the hydraulic conductivity of unsaturated soils. Soil Sci. Soc. Am. J. 44:892-898, 1980.

Vauclin, M., S. R. Vieira, G. Vachaud, and D. R. Nielsen, The use of cokriging with limited field soil observations, Soil Sci. Soc. Am. J., 47, 175-184, 1983.

Vesselinov, V. V., S. P. Neuman, W. A. Illman, and G. A. Zyvoloski, Three-dimensional inverse interpretation of pneumatic tests in unsaturated fractured rocks, XIII International Conference on Computational Methods in Water Resources, Calgary, Alberta, Canada, June 25-29, 2000.

Wang, J. S. Y., P. J. Cook, R. C. Trautz, R. Salve, A. L. James, S. Finsterle, T. K. Tokunaga, R. Solbau, J. Clyde, A. L. Flint, and L. E. Flint, Field Testing And Observation of Flow Paths In Niches: Phase 1 Status Report Of The Drift Seepage Test And Niche Moisture Study, Level 4 milestone SPC314M4 for WBS 1.2.3.3.1.2.4, Yucca Mountain Site Characterization Project, 1998.

Watson, K. K., An instantaneous profile method for determining the hydraulic conductivity unsaturated porous materials, Water Resour. Res., 2, 709-715, 1966.

Yates, S. R., and A. W. Warrick, Estimating soil water content using cokriging, Soil Sci. Soc. Am. J., 51, 23-30, 1987.

Yeh, W. W-G., Review of parameter identification procedures in groundwater hydrology: The inverse problem, Water Resour. Res., 22(2), 95-108, 1986.

Yeh, T. C., T. C. Rasmussen, and D. D. Evans, Simulation of Liquid and Vapor Movement in Unsaturated Fractured Rock at the Apache Leap Tuff Site - Models and Strategies, NUREG/CR5097, U. S. Nuclear Regulatory Commission, Washington D. C., 1988.

Yeh, T.-C. J., One-dimensional steady-state infiltration in heterogeneous soils, Water Resour. Res., 25(10), 2149-2158, 1989.

Yeh, T.-C. J., A.L. Gutjahr, and M. Jin, An iterative cokriging-like technique for groundwater flow modeling, Ground Water, 33(1), 33-41, 1995. 
Yeh, T.-C. J., and J. Zhang, A geostatistical inverse method for variably saturated flow in the vadose zone, Water Resour. Res., 32(9), 2757-2766, 1996.

Yeh, T.-C. J., Scale issues of heterogeneity in vadose-zone hydrology, in Scale Dependence and Scale Invariance in Hydrology, edited by G. Sposito, Cambridge Press, 1998.

Yeh, T.-C. J., and S. Liu, Hydraulic tomography: Development of a new aquifer test method, Water Resour. Res., 36(8), 2095-2105, 2000.

Yeh, T.-C., M. Jin, and S. Hanna, An iterative stochastic inverse method: conditional effective transmissivity and hydraulic head fields, Water Resour. Res., 32(1), 85-92, 1996.

Yeh, T.-C. J, S. Liu, R. J. Glass, K. Baker, J. R. Brainard, D. Alumbaugh, and D. LaBrecque , A Geostastically-Based Inverse Model for Electrical Resistivity Surveys and Its Applications to Vadose Zone Hydrology, Water Resources Research, in review, 2002.

Zhang, J. and T.-C. J. Yeh, An iterative geostatistical inverse method for steady flow in the vadose zone, Water Resour. Res., 33(1), 63-71, 1997.

Zurmühl, T., Evaluation of different boundary conditions for independent determination of hydraulic parameters using outflow methods, in W. Durner, J. Halbertsma, and M. Cislerova (eds.) Advanced Methods to Determine Hydraulic Properties of Soils, Dept. of Hydrology University of Bayreuth, Germany, 81-84, 1996. 


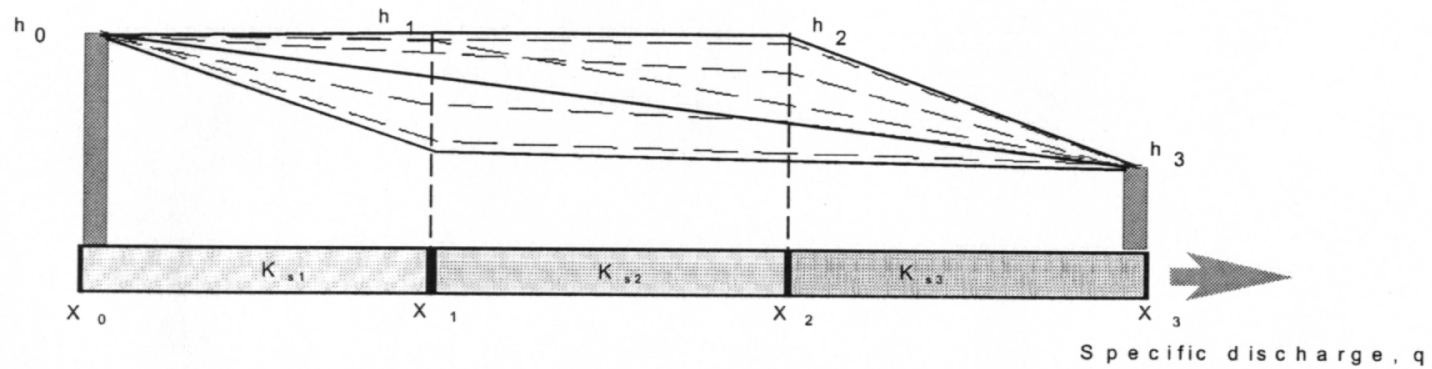

Figure 1. Possible head distributions along a heterogeneous soil column. 


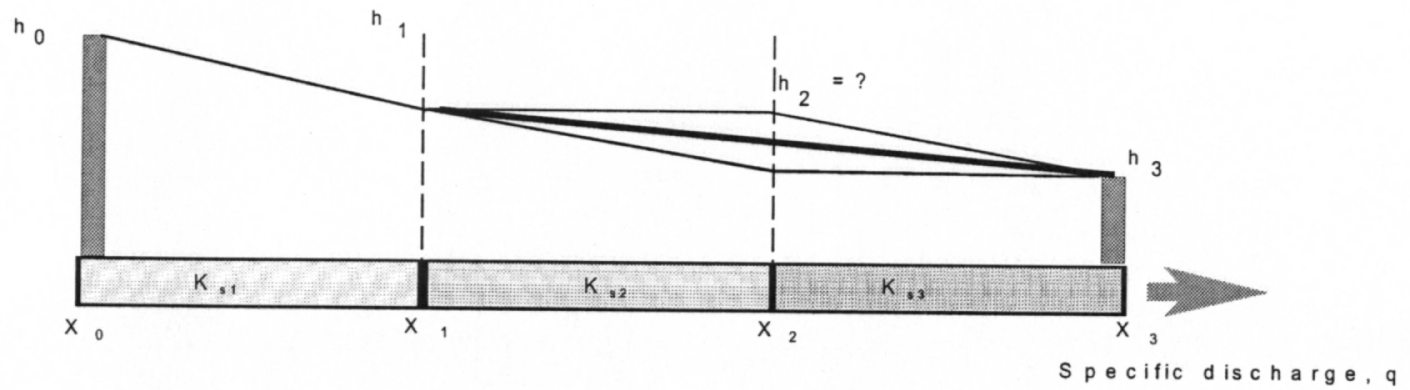

Figure 2. Possible head distributions along a heterogeneous column if an additional $h_{1}$ value is specified. 


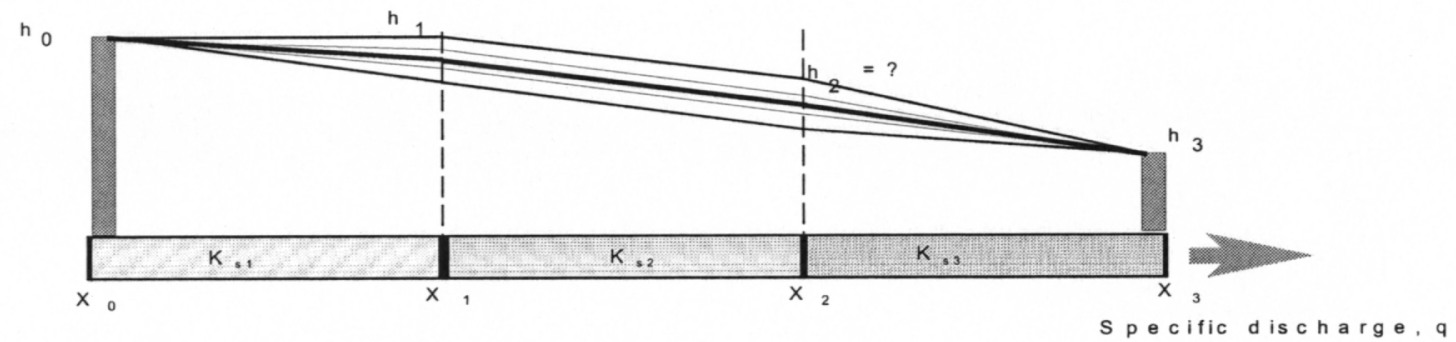

Figure 3. Possible head distributions along a heterogeneous column if $K_{s 2}$ is given. 

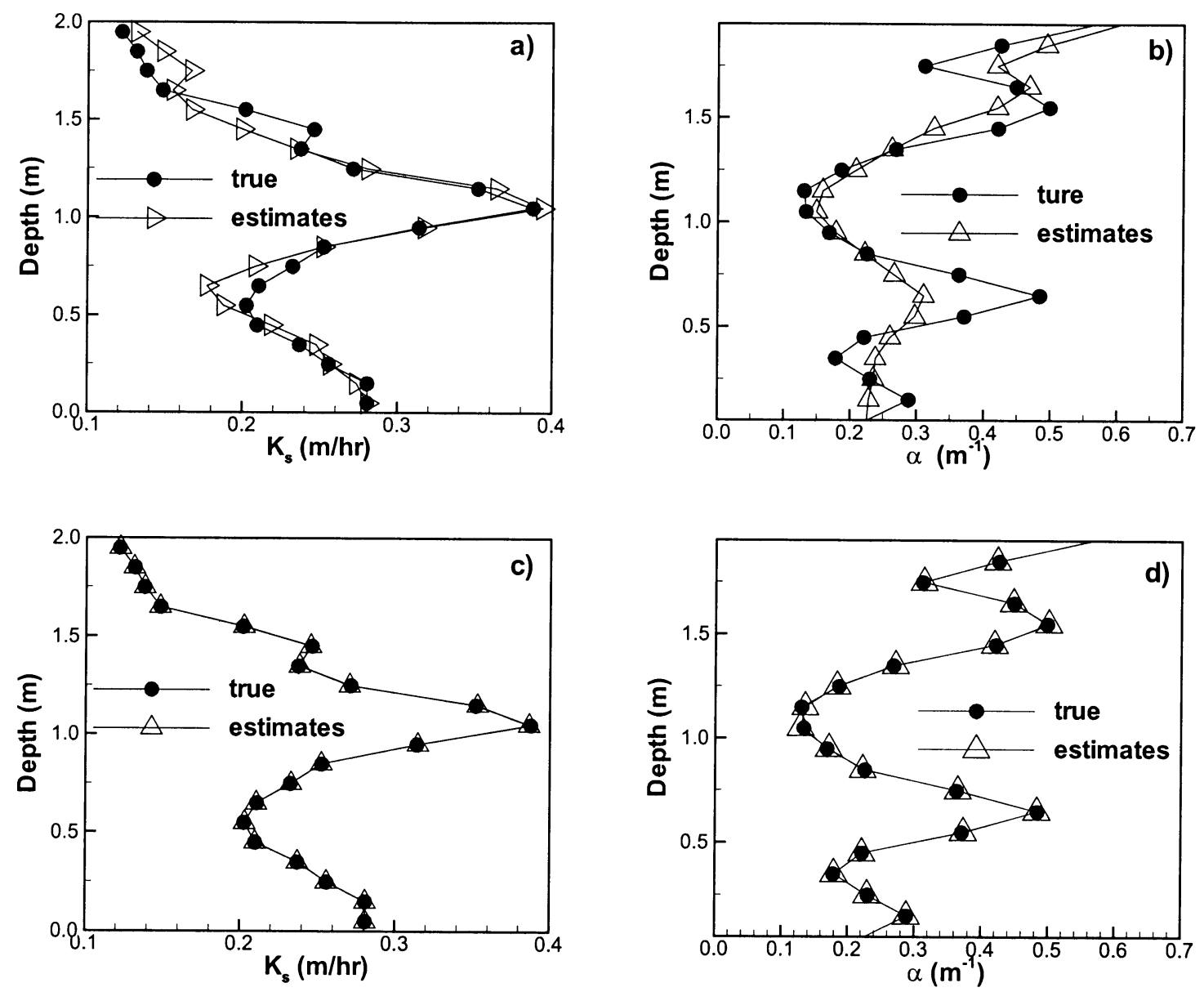

Figure 4. Comparisons of true and the estimated $\mathrm{K}_{\mathrm{s}}$ and $\alpha$ values (after removing their means) from one flux boundary condition, a) and b), respectively. Figure $4 \mathrm{c}$ ) and d) compare the true and estimated values for two flux boundary conditions. 


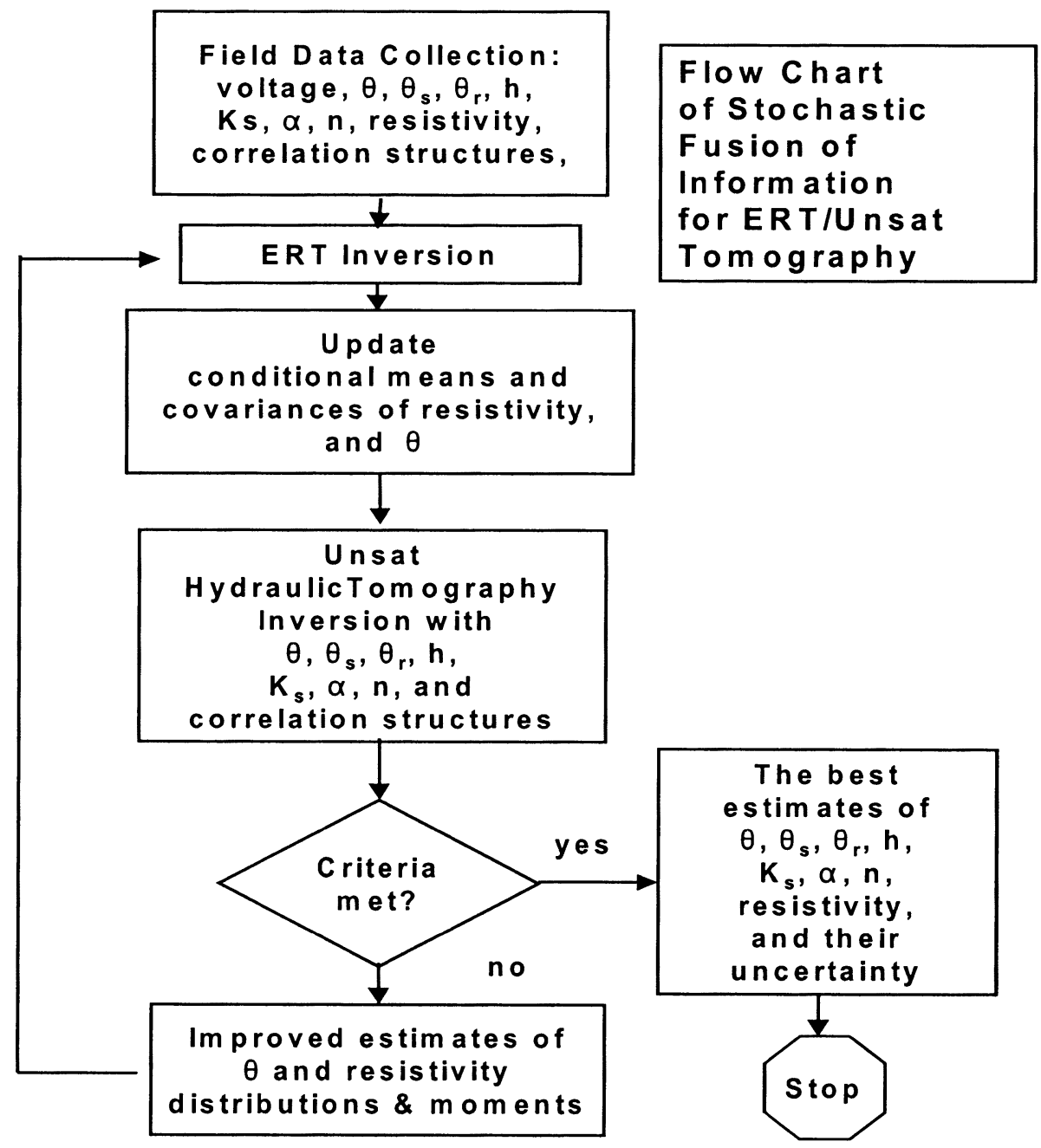

Figure 5. Level 2 iterative stochastic fusion algorithm. 
(A) True $\alpha$ Field

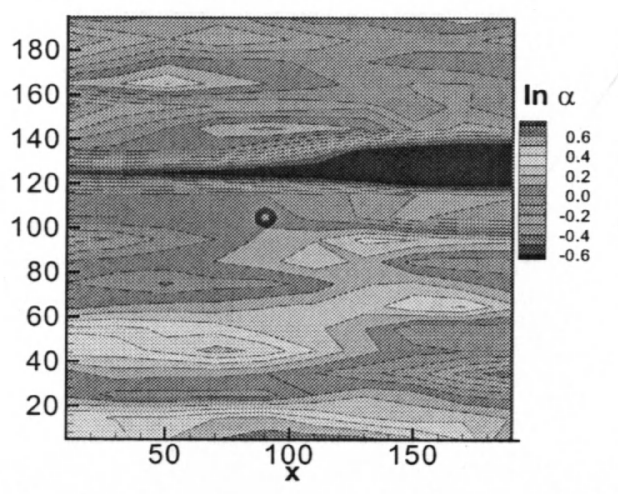

(B) True moisture content ( $\theta)$

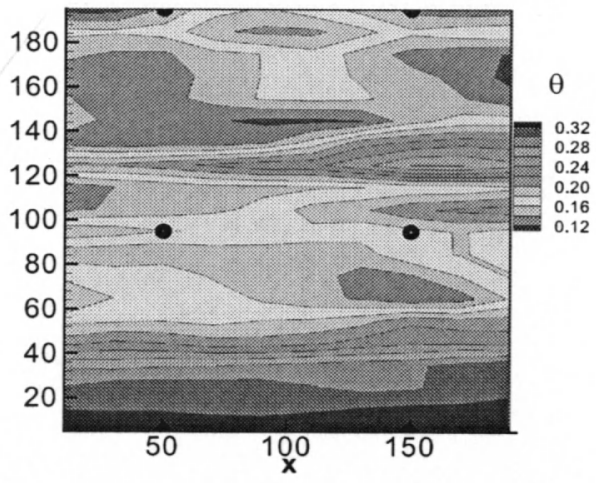

Figure 6. True $\alpha$ and $\theta$ fields. 

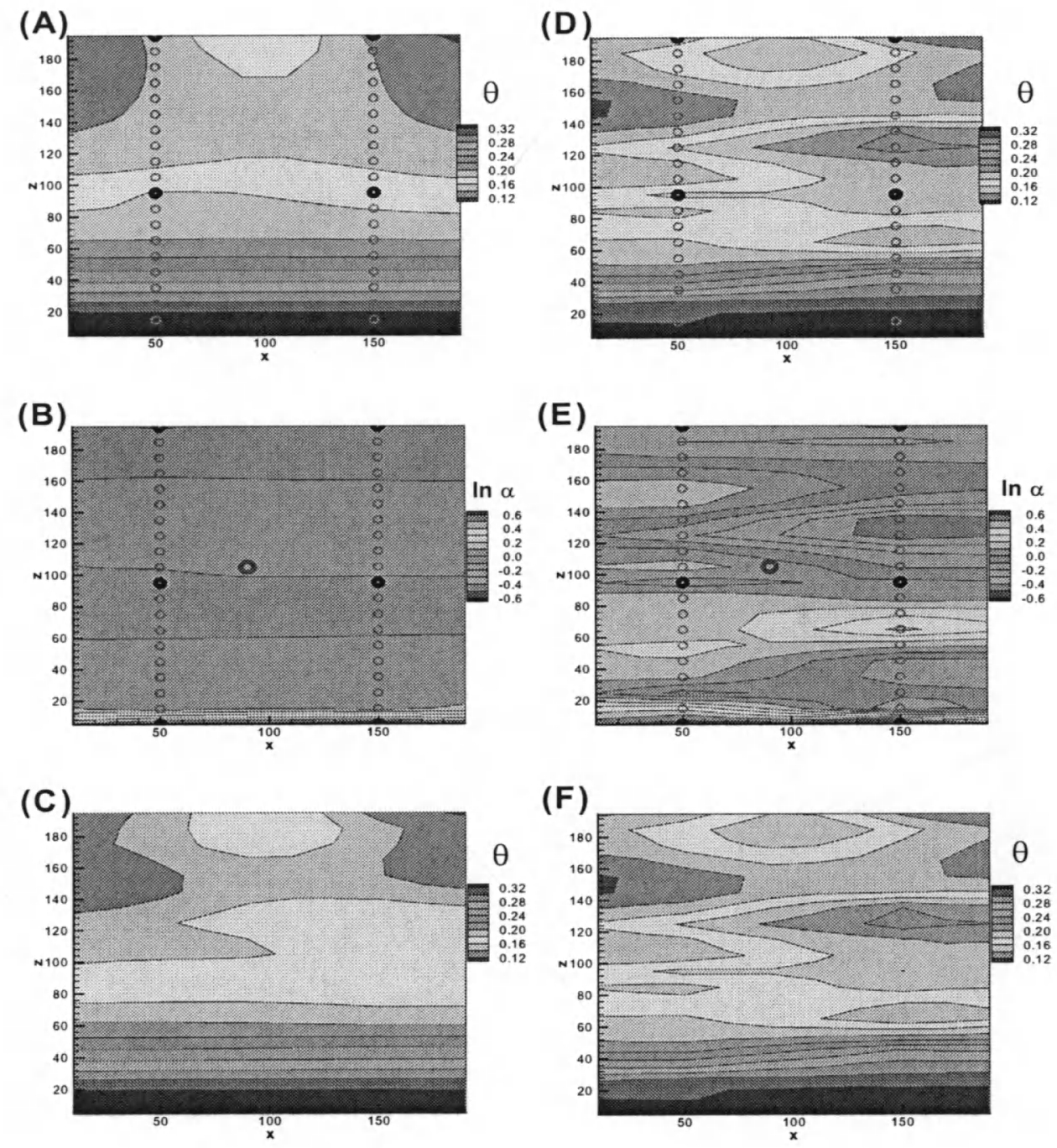

Figure 7. Estimated $\alpha$ and $\theta$ fields using the two approaches. 